TRANSACTIONS OF THE

AMERICAN MATHEMATICAL SOCIETY

Volume 350, Number 2, February 1998, Pages 429-472

S $0002-9947(98) 01673-0$

\title{
STABILITY OF MULTIPLE-PULSE SOLUTIONS
}

\author{
BJÖRN SANDSTEDE
}

\begin{abstract}
In this article, stability of multiple-pulse solutions in semilinear parabolic equations on the real line is studied. A system of equations is derived which determines stability of $N$-pulses bifurcating from a stable primary pulse. The system depends only on the particular bifurcation leading to the existence of the $N$-pulses.

As an example, existence and stability of multiple pulses are investigated if the primary pulse converges to a saddle-focus. It turns out that under suitable assumptions infinitely many $N$-pulses bifurcate for any fixed $N>$ 1. Among them are infinitely many stable ones. In fact, any number of eigenvalues between 0 and $N-1$ in the right half plane can be prescribed.
\end{abstract}

\section{INTRODUCTION}

This article is concerned with the stability of travelling waves of semilinear parabolic equations on the real line. Consider

$$
U_{t}=\mathcal{A} U+F(U, \varepsilon),
$$

where $U(x, t) \in \mathbb{R}^{m}$ and $x, t \in \mathbb{R}$ are space and time, respectively. The operator $\mathcal{A}$ is diagonal, the entries of which are homogeneous linear differential operators, defining an analytic semigroup on $B U\left(\mathbb{R}, \mathbb{R}^{m}\right)$, while the Nemitskii-operator $F$ maps certain fractional power spaces associated with $\mathcal{A}$ smoothly into themselves, see [Hen81]. A particular example is the system

$$
U_{t}=D U_{x x}+G(U)
$$

where $D$ is a diagonal $m \times m$ matrix and $G: \mathbb{R}^{m} \rightarrow \mathbb{R}^{m}$ denotes a smooth function. We are interested in pulse solutions of (1.1) which are travelling waves $U(\xi)=$ $U(x-c t)$ of $(1.1)$ decaying exponentially to the same constant vector $\bar{U}$, that is,

$$
\lim _{\xi \rightarrow \pm \infty} U(\xi)=\bar{U}
$$

Transforming (1.1) into a moving coordinate frame $(x, t) \mapsto(x-c t, t)=(\xi, t)$ yields

$$
U_{t}=\mathcal{A} U+c U_{\xi}+F(U, \varepsilon),
$$

where $\mathcal{A}$ operates on $U(\xi)$ as before on $U(x)$. Therefore, a pulse solution $U$ of (1.1) travelling with speed $c$ corresponds to a steady state of (1.2)

$$
\mathcal{A} U+c U_{\xi}+F(U, \varepsilon)=0 .
$$

Received by the editors April 25, 1995 and, in revised form, September 19, 1995.

1991 Mathematics Subject Classification. Primary 35B35, 58F14, 34C37.

Key words and phrases. Partial differential equations, solitary waves, homoclinic orbits, stability.

(C)1998 American Mathematical Society 
In order to investigate the stability properties of a given pulse $U$, two problems do arise. The first is whether linear stability implies nonlinear stability; the second one is concerned with the linear stability, that is, the investigation of the spectrum of the linearization of (1.3) at the pulse $U$. We will only consider situations where the first problem is solved. Often, one can use center manifold theory to conclude this property, see e.g. [Hen81], [BJ89] or [AJ94] and the references therein. For the second problem, one has to investigate the spectrum of the operator

$$
\mathcal{A} V+c V_{\xi}+D_{U} F(U, \varepsilon) V .
$$

The spectrum decomposes into the essential and the normal part. The latter consists of all isolated eigenvalues of finite multiplicity, while the former is the complement of this set. We assume that the essential spectrum is strictly contained in the left half plane. Again this situation arises frequently in applications. Indeed, the essential spectrum is determined by the spectrum of the steady state $\bar{U}$ alone, see [Hen81, Theorem A.2]. If the steady state is stable, the essential spectrum of the pulse has negative real part.

Under these two basic assumptions, the stability problem of a pulse reduces to determine the eigenvalues - isolated ones with finite multiplicity - of the linearization, that is, solutions of the equation

$$
\mathcal{A} V+c V_{\xi}+D_{U} F(U, \varepsilon) V=\lambda V .
$$

Note that (1.3) and (1.5) are ordinary differential equations in the variable $\xi$.

Suppose now that for $(\varepsilon, c)=\left(\varepsilon_{1}, c_{1}\right)$ a pulse $U_{1}$ of (1.3) exists. Under certain conditions, equation (1.3) admits $N$-pulse solutions $U_{N}$ for values of $(\varepsilon, c)$ close to $\left(\varepsilon_{1}, c_{1}\right)$. These $N$-pulses look like $N$ copies of the primary pulse $U_{1}$ widely spaced in $\xi$. Throughout, we will assume that the primary pulse $U_{1}$ is asymptotically stable. The question addressed in the present paper is the stability of the bifurcating $N$ pulses.

By the results of [AGJ90] and the basic assumptions stated above, the spectrum of (1.4) evaluated at the $N$-pulse $U_{N}$ is contained in the left half plane with the possible exception of eigenvalues close to zero and eigenvalues bifurcating at infinity. Indeed, this follows from the relation

$$
c_{1}(\mathcal{E}(K))=W(D(K))
$$

proved in [AGJ90] for general eigenvalue problems of the form (1.5). The nonexistence of eigenvalues with positive real part and large modulus can be deduced from the linearization (1.5) being sectorial provided there are bounds independent of $\varepsilon$ on the lower order terms, see e.g. [AGJ90, Proposition 2.2] for systems of second order. Therefore, the relevant problem is to determine the eigenvalues close to zero. As a consequence of Theorem 2 and Rouché's theorem their number is equal to $N$. A more geometric proof of this fact can be obtained by applying the results of [AGJ90], [GJ90]. Indeed, the Chern number $c_{1}$ appearing in (1.6) is additive and thus the winding number of the Evans function $D$ associated with the $N$-pulse with respect to zero is $N$, see [AJ94]. One of these $N$ eigenvalues is equal to zero with eigenfunction $U_{N}^{\prime}$. The problem investigated here is the computation of the remaining $N-1$ eigenvalues close to zero. 
To this end, we rewrite (1.3) and (1.5) as ordinary differential equations of first order

$$
\begin{aligned}
& \dot{u}=f(u, \mu), \quad u \in \mathbb{R}^{n}, \mu=(\varepsilon, c), \\
& \dot{v}=\left(D_{u} f(u, \mu)+\lambda B\right) v,
\end{aligned}
$$

where $B$ is an $n \times n$ matrix. We have to solve (1.8) evaluated at an $N$-pulse $q_{N}$ of (1.7) for $\lambda$ and $v$ such that $\lambda \in \mathbb{C}$ is close to zero and $v$ is a nonzero eigenfunction bounded on $\mathbb{R}$. Theorem 2 describes the eigenvalues $\lambda$ of (1.8) close to zero by means of zeroes of the function $E(\lambda)=\operatorname{det} S(\lambda)$ where the $N \times N$ matrix $S(\lambda)$ is given by

$$
S(\lambda)=A-M \lambda+\text { h.o.t. }
$$

for some $M \in \mathbb{R}$. The entries of $A$ are determined by the particular bifurcation scenario leading to the existence of the $N$-pulse $q_{N}$ from a given primary pulse $q_{1}$. The method used to obtain this reduction was originally introduced in [Lin90]. In [San93], it was further developed such that bifurcations leading to $N$-pulses could be handled. The matrix $A$ is most easily computed if the existence proof of the $N$-pulses is done using Lin's method as presented in [San93]. In Lemma 4.1, we relate the order of a zero of $E(\lambda)$ to the algebraic multiplicity of the eigenvalue $\lambda$ of the original PDE operator (1.4). Finally, Lemma 5.2 shows how to solve the reduced equation $E(\lambda)=0$ by computing the eigenvalues of the matrix $A$.

As an application, we consider a stable primary homoclinic solution to a saddlefocus. It will be shown that infinitely many $N$-pulses bifurcate for any $N>1$ under certain hypotheses. Moreover, we characterize the stability of these $N$-pulses. It turns out that any number of unstable eigenvalues between 0 and $N-1$ occurs for infinitely many of the $N$-pulses. In particular, infinitely many stable $N$-pulses bifurcate for any $N$.

Let us mention related results. In [EFF82], the existence of double pulses in nerve-axon equations was shown, while [YM89] solved the stability problem associated with these double pulses. Here, the primary pulse converges to a saddle-focus possessing a one-dimensional unstable manifold. In the same setting, [Fer86] proved the existence of infinitely many $N$-pulses for any $N$, see also [Gas83]. If the dimensions of stable and unstable manifolds are both equal to two and the eigenvalues of the equilibrium have nonvanishing imaginary part, existence of multiple pulses was shown by [Gle89]. Both existence and stability of double pulses are investigated in[AJ94] in this case. Under an additional assumption, [AJ93] proved the existence of stable triple pulses. A general theory of the Evans function was developed in [AGJ90]. Stability of 1-pulses and 3-fronts bifurcating from a heteroclinic cycle is investigated in [Nii95a] and [Nii95b], respectively. In [EMS90] and [Mer92], conditions for the stability of $N$-pulses for any $N$ are given based on a formal analysis. Actually, they formally derived an ordinary differential equation in $\mathbb{R}^{N}$ which describes the dynamics of all $N$-pulse like solutions with arbitrarily, widely spaced humps. In particular, determining equilibria of the ODE together with their stability with respect to the ODE corresponds to deriving conditions on the PDE stability of $N$-pulses. The resulting condition seems to differ from the one given here in the way the bounded solution $\psi_{1}$ of the adjoint variational equation enters, compare e.g. [EMS90, (3.19)] with Theorem 2.

The paper is organized as follows. In Section 2, we state the basic assumptions and the main results concerning the ODE's (1.7) and (1.8), the proofs of which 
are contained in Section 3. The multiplicity lemma is proved in Section 4. We provide some basic strategies for solving the resulting reduced equations appearing in the main result and give relations to the Evans function in Section 5. Finally, in Section 6, we apply our results to a homoclinic orbit to a saddle-focus proving existence and stability of $N$-pulses. Section 7 is devoted to a discussion.

\section{ACKNOWLEDGEMENT}

I am very grateful to Christopher K. R. T. Jones for many stimulating discussions and for introducing me to this subject. Moreover, I wish to thank Reiner Lauterbach and Daniela Peterhof for helpful comments on an earlier draft.

\section{The EIGENVAlue PROBlem For the VARIATIONAL EQUATION}

Consider

$$
\dot{u}=f(u, \mu), \quad(u, \mu) \in \mathbb{R}^{n} \times \mathbb{R}^{p},
$$

with $f$ being smooth. We assume that zero is a hyperbolic equilibrium for $\mu=\mu_{1}$. Hence, there exist real numbers $\alpha^{s}, \alpha^{u}>0$ such that

(H1) $\operatorname{Re} \sigma\left(D_{u} f\left(0, \mu_{1}\right)\right) \subset\left(-\infty,-\alpha^{s}\right) \cup\left(\alpha^{u}, \infty\right)$.

Let $E^{s}$ and $E^{u}$ be the generalized eigenspaces associated with the spectral sets appearing in (H1) and denote the corresponding projections by $P_{0}^{s}$ and $P_{0}^{u}$, respectively. We denote by $p(\mu)$ the family of equilibria of (2.1) satisfying $p\left(\mu_{1}\right)=0$.

Suppose that for $\mu=\mu_{1}$ a pulse solution $q_{1}(t)$ converging to zero for $t \rightarrow \pm \infty$ exists. We assume that $q_{1}$ is nondegenerate:

(H2) $T_{q_{1}(0)} W^{s}\left(0, \mu_{1}\right) \cap T_{q_{1}(0)} W^{u}\left(0, \mu_{1}\right)=\mathbb{R} \dot{q}(0)$.

Here, $W^{s}$ and $W^{u}$ denote stable and unstable manifolds, respectively. (H2) is equivalent to the fact that $\dot{q}_{1}(t)$ is the unique bounded solution of the variational equation

$$
\dot{v}=D_{u} f\left(q_{1}(t), \mu_{1}\right) v .
$$

Then the adjoint variational equation

$$
\dot{w}=-D_{u} f\left(q_{1}(t), \mu_{1}\right)^{*} w
$$

possesses a unique bounded solution, too, which we denote by $\psi_{1}(t)$, see [San93]. The solution $\psi_{1}(t)$ satisfies

$$
\psi_{1}(t) \perp T_{q_{1}(t)} W^{s}\left(0, \mu_{1}\right)+T_{q_{1}(t)} W^{u}\left(0, \mu_{1}\right)
$$

for all $t \in \mathbb{R}$. Next we decompose the tangent space at $q_{1}(0)$ according to

$$
\mathbb{R}^{n}=\mathbb{R} \psi_{1}(0) \oplus \mathbb{R} \dot{q}_{1}(0) \oplus Y^{+} \oplus Y^{-},
$$

where $Y^{+}$and $Y^{-}$are chosen such that

$$
\begin{aligned}
& \mathbb{R} \dot{q}_{1}(0) \oplus Y^{+}=T_{q_{1}(0)} W^{s}\left(0, \mu_{1}\right), \\
& \mathbb{R} \dot{q}_{1}(0) \oplus Y^{-}=T_{q_{1}(0)} W^{u}\left(0, \mu_{1}\right)
\end{aligned}
$$

Then we have the following two lemmata; see also Figure 1. 


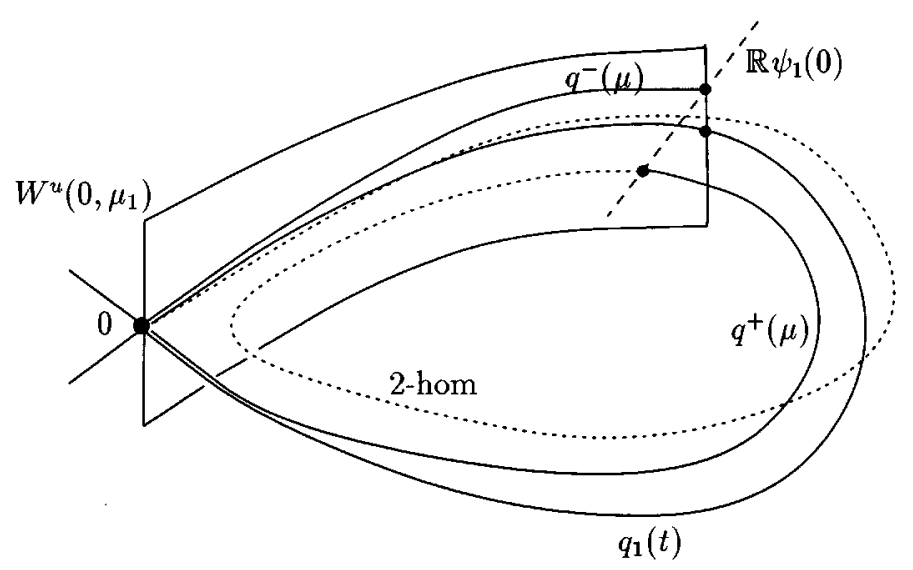

Figure 1. A 2-homoclinic solution

Lemma 2.1 ([San93, Lemma 3.6]). There exists a smooth change of coordinates such that $p(\mu)=0$ holds. Moreover, assuming $p(\mu)=0, W_{l o c}^{s}(0, \mu) \subset E^{s}, W_{l o c}^{u}(0, \mu)$ $\subset E^{u}$ and

$$
\begin{aligned}
& W^{s}(0, \mu) \cap U_{\delta}\left(q_{1}(0)\right) \subset q_{1}(0)+\mathbb{R} \dot{q}_{1}(0) \oplus Y^{+}, \\
& W^{u}(0, \mu) \cap U_{\delta}\left(q_{1}(0)\right) \subset q_{1}(0)+\mathbb{R} \dot{q}_{1}(0) \oplus Y^{-},
\end{aligned}
$$

are satisfied for some $\delta>0$.

We assume that coordinates are changed according to Lemma 2.1.

Lemma 2.2 ([San93, Lemma 3.3]). There exist smooth families of solutions $q^{+}(\mu)$ and $q^{-}(\mu)$ for $\mu$ close to $\mu_{1}$ such that

$$
\begin{array}{ll}
q^{+}(\mu)(t) \in W^{s}(0, \mu), & t \geq 0, \\
q^{-}(\mu)(t) \in W^{u}(0, \mu), & t \leq 0,
\end{array}
$$

and

$$
\begin{aligned}
q^{+}(\mu)(0)-q^{-}(\mu)(0) & \in \mathbb{R} \psi_{1}(0), \\
q^{ \pm}(\mu)(0)-q_{1}(0) & \in \mathbb{R} \psi_{1}(0) \oplus Y^{+} \oplus Y^{-}, \\
q^{ \pm}\left(\mu_{1}\right)(0) & =q_{1}(0) .
\end{aligned}
$$

The families $q^{ \pm}(\mu)$ are unique with respect to these properties. Moreover, there is a unique bounded solution $\psi(\mu)$ of

$$
\dot{w}=-D_{u} f(\tilde{u}(\mu), \mu)^{*} w
$$

with $\psi(\mu)(0)=\psi_{1}(0)$. Here, $\tilde{u}$ is defined by $\tilde{u}(\mu)(t)=q^{-}(\mu)(t)$ for $t<0$ and $\tilde{u}(\mu)(t)=q^{+}(\mu)(t)$ for $t>0$. In addition, $\psi\left(\mu_{1}\right)(t)=\psi_{1}(t)$ holds.

Now we are in a position to characterize $N$-pulses which exist for parameter values $\mu$ close to $\mu_{1}$ and which are close to the trace of the primary pulse $q_{1}$ in phase space. Note that [San93] contains a sharper version of Theorem 1.

Let $U_{\delta}\left(q_{1}\right)$ denote the neighborhood of the orbit $\left\{q_{1}(t) \mid t \in \mathbb{R}\right\}$ of size $\delta$. The supremum norm is denoted by $\|\cdot\|$. 
Theorem 1 ([San93]). There exist $\delta_{0}, \delta_{1}>0$ small with the following property.

Suppose that $q_{N}(t)$ is a pulse solution of (2.1) satisfying $q_{N}(t) \in U_{\delta_{0}}\left(q_{1}\right)$ for all $t \in \mathbb{R}$ and existing for a parameter value $\mu_{N}$ with $\left|\mu_{N}-\mu_{1}\right|<\delta_{0}$. Moreover, assume that $q_{N}(t)$ intersects the transverse section

$$
\left\{q_{1}(0)+x \mid x \in \mathbb{R} \psi_{1}(0) \oplus Y^{+} \oplus Y^{-} \text {and }|x| \leq \delta_{0}\right\}
$$

$N$ times. Throughout, let $i=1, \ldots, N$ and $j=1, \ldots, N-1$. Then there exist unique numbers $T_{j}>\frac{1}{\delta_{1}}$ for $j=1, \ldots, N-1$ and unique functions

$$
u_{i}^{-}:\left[-T_{i-1}, 0\right] \rightarrow \mathbb{R}^{n}, \quad u_{i}^{+}:\left[0, T_{i}\right] \rightarrow \mathbb{R}^{n}
$$

for $i=1, \ldots, N$ with $T_{0}=T_{N}=\infty$ such that:

$$
\begin{aligned}
q^{-}(0)+u_{i}^{-}(0) & =q^{+}(0)+u_{i}^{+}(0), \\
q^{-}\left(-T_{j}\right)+u_{j+1}^{-}\left(-T_{j}\right) & =q^{+}\left(T_{j}\right)+u_{j}^{+}\left(T_{j}\right)
\end{aligned}
$$

and $q_{N}(t)$ is piecewise defined via

$$
q_{N}\left(t+2 \sum_{k=1}^{i-1} T_{k}\right)= \begin{cases}q^{-}(t)+u_{i}^{-}(t) & \text { for } t \in\left(-T_{i-1}, 0\right], \\ q^{+}(t)+u_{i}^{+}(t) & \text { for } t \in\left(0, T_{i}\right] .\end{cases}
$$

Here, $q^{ \pm}$is evaluated at $\mu=\mu_{N}$. Moreover, the estimates

$$
\left\|u_{i}\right\| \leq C \sup _{t \geq T}\left(\left|q^{+}\left(\mu_{N}\right)(t)\right|+\left|q^{-}\left(\mu_{N}\right)(-t)\right|\right),
$$

$$
\begin{aligned}
& \text { (ii) }\left|u_{j+1}^{-}\left(-T_{j}\right)-q^{+}\left(\mu_{N}\right)\left(T_{j}\right)\right| \leq C e^{-\alpha T} \sup _{t \geq T}\left(\left|q^{+}\left(\mu_{N}\right)(t)\right|+\left|q^{-}\left(\mu_{N}\right)(-t)\right|\right), \\
& \text { (iii) }\left|u_{j}^{+}\left(T_{j}\right)-q^{-}\left(\mu_{N}\right)\left(-T_{j}\right)\right| \leq C e^{-\alpha T} \sup _{t \geq T}\left(\left|q^{+}\left(\mu_{N}\right)(t)\right|+\left|q^{-}\left(\mu_{N}\right)(-t)\right|\right)
\end{aligned}
$$

hold with $T=\min _{j=1, \ldots, N-1} T_{j}$ and $\alpha=\min \left(\alpha^{s}, \alpha^{u}\right)$. The constant $C$ depends only on $N, \delta_{0}$ and $\delta_{1}$.

Proof. The statements of the theorem are consequences of [San93, Section 3]. The estimate (2.6)(i) follows from [San93, (3.38), (3.39) and (3.42)] and the definition $[$ San93, (3.17)]. The other two estimates follow from the proof of [San93, Lemma 3.20]. Indeed, in the notation of [San93], we have

$$
\begin{aligned}
& w_{i}\left(\omega_{i+1}\right)=w_{i}^{s}\left(\omega_{i+1}\right)+d_{i+1}^{u}+R_{i+1}^{u}, \\
& \hat{w}_{i}\left(-\omega_{i}\right)=\hat{w}_{i}^{u}\left(-\omega_{i}\right)+d_{i}^{s}+R_{i}^{s}
\end{aligned}
$$

and the estimates for $R_{i}^{s}$ and $R_{i}^{u}$ can be written as

$$
\|R\| \leq C e^{-\alpha T}\|d\|
$$

see [San93, Lemma 3.20].

The stability of the pulses described in Theorem 1 is considered in the next theorem, which is the main result of the present article. Assume

$$
B: \mathbb{R}^{n} \times \mathbb{R}^{p} \rightarrow \mathbb{R}^{n \times n}
$$

is a smooth function. We are interested in bounded solutions $v$ of

$$
\dot{v}=D_{u} f\left(q_{N}(t), \mu_{N}\right) v+\lambda B\left(q_{N}(t), \mu_{N}\right) v
$$

for $\lambda \in \mathbb{C}$, where $q_{N}$ is an $N$-pulse existing for the parameter value $\mu_{N}$. However, we applied a change of coordinates to (2.1). The consequences of this transformation for (2.7) are given in the next lemma, the proof of which is straightforward. 
Lemma 2.3. Consider the equation $\dot{u}=f(u, \mu)$ and change coordinates according to the transformation $x=h(u, \mu)$ yielding $\dot{x}=g(x, \mu)$. Then the equations

$$
\begin{aligned}
\dot{v} & =\left(D_{u} f(u, \mu)+\lambda B(u, \mu)\right) v, \\
\dot{w} & =-D_{u} f(u, \mu)^{*} w
\end{aligned}
$$

transform by the change $y=D_{u} h(u, \mu) v$ and $z=\left(D_{u} h(u, \mu)^{*}\right)^{-1} w$ into the system

$$
\begin{aligned}
& \dot{y}=\left(D_{x} g(x, \mu)+\lambda \widetilde{B}(x, \mu)\right) y, \\
& \dot{z}=-D_{x} g(x, \mu)^{*} z
\end{aligned}
$$

for some function $\widetilde{B}$. In addition, $M=\int_{-\infty}^{\infty}\left\langle\psi_{1}(t), B\left(q_{1}(t), \mu_{1}\right) \dot{q}_{1}(t)\right\rangle d t$ does not depend on the coordinate system chosen.

Therefore, the solution set of (2.7) remains unchanged if we transform (2.7) according to the lemma.

Theorem 2. There exists a $\delta_{2}>0$ small with the following property.

Let $\left(q_{N}, \mu_{N}\right)$ denote an $N$-pulse as described in Theorem 1 with associated return times $T_{j}$ for $j=1, \ldots, N-1$ and $T_{0}=T_{N}=\infty$. Then there exists a bounded nonzero solution $v$ of $(2.7)$ for $\lambda \in U_{\delta_{2}}(0) \subset \mathbb{C}$ if and only if

$$
E(\lambda)=\operatorname{det} S(\lambda)=\operatorname{det}(A-M \lambda \mathrm{id}+R(\lambda))=0 .
$$

Here, the tridiagonal matrix $A=\left(a_{i j}\right)_{i, j=1, \ldots, N}$ is defined by

$$
A=\left(\begin{array}{ccccc}
-a_{1} & a_{1} & & & \\
-\tilde{a}_{1} & \tilde{a}_{1}-a_{2} & a_{2} & & \\
& -\tilde{a}_{2} & \tilde{a}_{2}-a_{3} & a_{3} & \\
& & \ddots & \ddots & \\
& & & -\tilde{a}_{N-1} & \tilde{a}_{N-1}
\end{array}\right)
$$

with

$$
\begin{aligned}
& a_{j}=\left\langle\psi\left(T_{j}\right), \dot{q}^{-}\left(-T_{j}\right)\right\rangle, \\
& \tilde{a}_{j}=\left\langle\psi\left(-T_{j}\right), \dot{q}^{+}\left(T_{j}\right)\right\rangle,
\end{aligned}
$$

where $\psi$ and $q^{ \pm}$are evaluated at the parameter value $\mu_{N}$. Moreover,

$$
M=\int_{-\infty}^{\infty}\left\langle\psi_{1}(t), B\left(q_{1}(t), \mu_{1}\right) \dot{q}_{1}(t)\right\rangle d t \in \mathbb{R} .
$$

The remainder term $R(\lambda)$ is analytic in $\lambda$ and can be estimated by

$$
\begin{aligned}
\|R(\lambda)\| \leq C\left(e^{-2 \alpha T} \sup _{t \geq T}\left(\left|q^{+}\left(\mu_{N}\right)(t)\right|+\left|q^{-}\left(\mu_{N}\right)(-t)\right|\right)\right. & \\
& \left.+|\lambda|\left(e^{-\alpha T}+\left|\mu_{N}-\mu_{1}\right|+|\lambda|\right)\right),
\end{aligned}
$$

with the notation introduced in Theorem 1.

In Appendix A, an improved estimate of the remainder term is given.

An interpretation of $M$ in terms of the Evans function associated with the primary pulse $q_{1}$ is given in Section 5. Note that $M$ does not depend on the actual coordinate system chosen due to Lemma 2.3. 


\section{THE PROOF}

The main idea for proving Theorem 2 consists in interpreting eigenfunctions of the linearized system (2.7) associated with an $N$-pulse $q_{N}$ as $N$-homoclinic solutions again. Indeed, it will turn out that eigenfunctions corresponding to eigenvalues close to zero look like $N$ copies of $\dot{q}_{1}(t)$. Therefore, they can be considered as $N$ homoclinic solutions with respect to $\dot{q}_{1}(t)$ in the same way as $q_{N}(t)$ is a multiple pulse with respect to $q_{1}(t)$. This leads to the idea of applying the theory developed to prove Theorem 1 to the nonautonomous equation (2.7).

To this end, we are going to reformulate the eigenvalue problem (2.7) in Section 3.1. Then we are essentially in the setting considered in [Lin90, Lemma 2.2 and Theorem 2.4]. However, since we need to exploit the special structure of (2.7), we will give full proofs in Section 3.2 instead of referring to [Lin90]. Another reason for proceeding in this way is that sharper estimates will be needed later on which will not follow from [Lin90] but require techniques developed in [San93]. The equations considered in 3.2 are of general type and we give the relation to the original system in Section 3.3.

3.1. The reformulation. We have to investigate bounded solutions $(v, \lambda)$ of

$$
\dot{v}=\left(D_{u} f\left(q_{N}(t), \mu_{N}\right)+\lambda B\left(q_{N}(t), \mu_{N}\right)\right) v
$$

for $t \in \mathbb{R}$, where $\left(q_{N}, \mu_{N}\right)$ is an $N$-pulse of

$$
\dot{u}=f(u, \mu)
$$

close to $\left(q_{1}, \mu_{1}\right)$. We are interested in $\lambda \in \mathbb{C}$ close to zero. By Theorem 1 , it is possible to describe $q_{N}$ according to

$$
q_{N}\left(t+2 \sum_{k=1}^{i-1} T_{k}\right)= \begin{cases}q^{-}\left(\mu_{N}\right)(t)+u_{i}^{-}(t) & \text { for } t \in\left(-T_{i-1}, 0\right], \\ q^{+}\left(\mu_{N}\right)(t)+u_{i}^{+}(t) & \text { for } t \in\left(0, T_{i}\right]\end{cases}
$$

where $i=1, \ldots, N$. Remember that $T_{0}=T_{N}=\infty$. Therefore, we can rewrite (3.1) in the equivalent form

$$
\begin{aligned}
\dot{v}_{i}^{-}=\left(D_{u} f\left(q^{-}\left(\mu_{N}\right)(t)+u_{i}^{-}(t), \mu_{N}\right)+\lambda B\left(q^{-}\left(\mu_{N}\right)(t)+u_{i}^{-}(t), \mu_{N}\right)\right) v_{i}^{-} \\
\quad \text { for } t \in\left(-T_{i-1}, 0\right), \\
\quad \quad \text { for } t \in\left(0, T_{i}\right), \\
\dot{v}_{i}^{+}=\left(D_{u} f\left(q^{+}\left(\mu_{N}\right)(t)+u_{i}^{+}(t), \mu_{N}\right)+\lambda B\left(q^{+}\left(\mu_{N}\right)(t)+u_{i}^{+}(t), \mu_{N}\right)\right) v_{i}^{+} \\
v_{i}^{-}(0)=v_{i}^{+}(0), \quad \\
v_{j}^{+}\left(T_{j}\right)=v_{j+1}^{-}\left(-T_{j}\right)
\end{aligned}
$$

for $i=1, \ldots, N$ and $j=1, \ldots, N-1$ considered as equations over the complex field. Then

$$
v\left(t+2 \sum_{k=1}^{i-1} T_{k}\right)= \begin{cases}v_{i}^{-}(t) & \text { for } t \in\left(-T_{i-1}, 0\right], \\ v_{i}^{+}(t) & \text { for } t \in\left(0, T_{i}\right]\end{cases}
$$

is a solution of (3.1) and vice versa.

Notation. The superscripts ${ }^{+}$and ${ }^{-}$always correspond to $t>0$ and $t<0$, respectively. Moreover, families $\left(x_{i}\right)$ will be abbreviated by $x$. Throughout, the indices $i$ and $j$ run through $i=1, \ldots, N$ and $j=1, \ldots, N-1$. All vector spaces will be considered over the complex field. Many different constants, all of which 
are independent of the family $\left(T_{i}\right)$ and the parameter $\mu$, will be denoted by $C$ in this section. We will often suppress the dependence of $q^{ \pm}(\mu)$ on the parameter $\mu$ by setting $q^{ \pm}=q^{ \pm}(\mu)$.

In order to exploit the fact that $q_{N}$ is a solution of (3.2) we employ the decomposition

$$
v_{i}^{ \pm}(t)=\left(\dot{q}^{ \pm}(t)+\dot{u}_{i}^{ \pm}(t)\right) d_{i}+w_{i}^{ \pm}(t)
$$

for $i=1, \ldots, N$ and arbitrary vectors $d=\left(d_{i}\right) \in \mathbb{C}^{N}$. Then $\left(d_{i}, w_{i}^{ \pm}\right)$solves

$$
\begin{aligned}
& \dot{w}_{i}^{ \pm}= D_{u} f\left(q^{ \pm}, \mu_{N}\right) w_{i}^{ \pm}+\lambda B\left(q^{ \pm}+u_{i}^{ \pm}, \mu_{N}\right)\left(\dot{q}^{ \pm}+\dot{u}_{i}^{ \pm}\right) d_{i} \\
&+\left(D_{u} f\left(q^{ \pm}+u_{i}^{ \pm}, \mu_{N}\right)-D_{u} f\left(q^{ \pm}, \mu_{N}\right)+\lambda B\left(q^{ \pm}+u_{i}^{ \pm}, \mu_{N}\right)\right) w_{i}^{ \pm} \\
& \text {on } t \in\left(-T_{i-1}, 0\right) \text { and } t \in\left(0, T_{i}\right), \text { respectively, } \\
& w_{i}^{-}(0)= w_{i}^{+}(0), \\
& w_{i}^{ \pm}(0) \in \mathbb{C} \psi_{1}(0) \oplus Y^{+} \oplus Y^{-}, \\
& w_{j}^{+}\left(T_{j}\right)=w_{j+1}^{-}\left(-T_{j}\right)+\left(\dot{q}^{-}\left(-T_{j}\right)+\dot{u}_{j+1}^{-}\left(-T_{j}\right)\right) d_{j+1}-\left(\dot{q}^{+}\left(T_{j}\right)+\dot{u}_{j}^{+}\left(T_{j}\right)\right) d_{j}
\end{aligned}
$$

if and only if $\left(v_{i}^{ \pm}\right)$solves (3.3). Indeed, if $\left(d_{i}, w_{i}^{ \pm}\right)$solve (3.6), then $\left(v_{i}^{ \pm}\right)$defined by (3.5) solve (3.3). On the other hand, suppose that $\left(v_{i}^{ \pm}\right)$is a solution of (3.3). Let $Q$ denote the projection onto $\mathbb{C} \dot{q}_{1}(0)$ with kernel $\mathbb{C} \psi_{1}(0) \oplus Y^{+} \oplus Y^{-}$. Define $d_{i}$ by

$$
d_{i}:=\frac{\left\langle\dot{q}_{1}(0), Q v_{i}^{+}(0)\right\rangle}{\left\langle\dot{q}_{1}(0), Q\left(\dot{q}^{+}\left(\mu_{N}\right)(0)+\dot{u}_{i}^{+}(0)\right)\right\rangle}
$$

and note that the denominator is nonzero. Then define $\left(w_{i}^{ \pm}\right)$according to (3.5) and observe that $\left(d_{i}, w_{i}^{ \pm}\right)$solve (3.6).

Note that $w_{i}^{ \pm}=0$ and $d_{i}=1$ for $i=1, \ldots, N$ is a bounded solution of (3.6) for $\lambda=0$ due to the fact that $q_{N}$ solves (3.2). In the following, we investigate the system

(i) $\dot{w}_{i}^{ \pm}=D_{u} f\left(q^{ \pm}\left(\mu_{N}\right)(t), \mu_{N}\right) w_{i}^{ \pm}+\left(G_{i}^{ \pm}(t)+\lambda B_{i}^{ \pm}(t)\right) w_{i}^{ \pm}+\lambda h_{i}^{ \pm}(t) d_{i}$,

(ii) $w_{i}^{ \pm}(0) \in \mathbb{C} \psi_{i}(0) \oplus Y^{+} \oplus Y^{-}$,

(iii) $w_{i}^{+}(0)-w_{i}^{-}(0) \in \mathbb{C} \psi_{i}(0)$,

(iv) $w_{j}^{+}\left(T_{j}\right)-w_{j+1}^{-}\left(-T_{j}\right)=D_{j} d$,

where we used the notations

$$
\begin{aligned}
G_{i}^{ \pm}(t) & =D_{u} f\left(q^{ \pm}\left(\mu_{N}\right)(t)+u_{i}^{ \pm}(t), \mu_{N}\right)-D_{u} f\left(q^{ \pm}\left(\mu_{N}\right)(t), \mu_{N}\right), \\
B_{i}^{ \pm}(t) & =B\left(q^{ \pm}\left(\mu_{N}\right)(t)+u_{i}^{ \pm}(t), \mu_{N}\right), \\
h_{i}^{ \pm}(t) & =B\left(q^{ \pm}\left(\mu_{N}\right)(t)+u_{i}^{ \pm}(t), \mu_{N}\right)\left(\dot{q}^{ \pm}\left(\mu_{N}\right)(t)+\dot{u}_{i}^{ \pm}(t)\right), \\
D_{j} d & =\left(\dot{q}^{-}\left(\mu_{N}\right)\left(-T_{j}\right)+\dot{u}_{j+1}^{-}\left(-T_{j}\right)\right) d_{j+1}-\left(\dot{q}^{+}\left(\mu_{N}\right)\left(T_{j}\right)+\dot{u}_{j}^{+}\left(T_{j}\right)\right) d_{j} .
\end{aligned}
$$

Note that a solution of (3.7) solves (3.6) if and only if

$$
\xi_{i}:=\left\langle\psi_{i}(0), w_{i}^{+}(0)-w_{i}^{-}(0)\right\rangle=0
$$

vanishes for $i=1, \ldots, N$, see also Lemma 3.6. Indeed, $w_{i}^{+}(0)-w_{i}^{-}(0) \in \mathbb{C} \psi_{1}(0)$ if $w$ solves $(3.7)$.

Thus, the question of determining the eigenvalues reduces to solving $\xi=0$ once (3.7) has been solved. For the functions defined in (3.8) we collect some estimates in the next lemma. 
Lemma 3.1. We have the estimates

$$
\begin{aligned}
& \left|G_{i}^{ \pm}(t)\right| \leq C\left|u_{i}^{ \pm}(t)\right| \leq C \sup _{t \geq T}\left(\left|q^{+}\left(\mu_{N}\right)(t)\right|+\left|q^{-}\left(\mu_{N}\right)(-t)\right|\right), \\
& \left|B_{i}^{ \pm}(t)\right| \leq C, \\
& \left|h_{i}^{ \pm}(t)-H(t)\right| \leq C\left(\left|\mu_{N}-\mu_{1}\right|+\sup _{t \geq T}\left(\left|q^{+}\left(\mu_{N}\right)(t)\right|+\left|q^{-}\left(\mu_{N}\right)(-t)\right|\right)\right), \\
& \quad D_{j} d=\left(\dot{q}^{+}\left(\mu_{N}\right)\left(T_{j}\right)+\dot{q}^{-}\left(\mu_{N}\right)\left(-T_{j}\right)\right)\left(d_{j+1}-d_{j}\right) \\
& \quad+O\left(e^{-\alpha T}|d| \sup _{t \geq T}\left(\left|q^{+}\left(\mu_{N}\right)(t)\right|+\left|q^{-}\left(\mu_{N}\right)(-t)\right|\right)\right)
\end{aligned}
$$

using the definition $H(t)=B\left(q_{1}(t), \mu_{1}\right) \dot{q}_{1}(t)$.

Proof. The first estimate follows from Theorem 1, (2.6)(i), the last one from (2.6)(ii) and (iii). Moreover, the third inequality is a consequence of Lemma 2.2 and again (2.6)(i). The second inequality is trivial.

3.2. The reduction. Consider the general system

(i) $\dot{w}_{i}^{ \pm}=D_{u} f\left(q^{ \pm}(\mu)(t), \mu\right) w_{i}^{ \pm}+\left(G_{i}^{ \pm}(t)+\lambda B_{i}^{ \pm}(t)\right) w_{i}^{ \pm}+\lambda h_{i}^{ \pm}(t) d_{i}$,

(ii) $w_{i}^{ \pm}(0) \in \mathbb{C} \psi_{i}(0) \oplus Y^{+} \oplus Y^{-}$,

(iii) $w_{i}^{+}(0)-w_{i}^{-}(0) \in \mathbb{C} \psi_{1}(0)$,

(iv) $w_{j}^{+}\left(T_{j}\right)-w_{j+1}^{-}\left(-T_{j}\right)=D_{j} d$,

for arbitrary functions $G=\left(G_{i}^{ \pm}\right), h=\left(h_{i}^{ \pm}\right)$, matrices $D=\left(D_{j}\right)$ and $\lambda \in \mathbb{C}$ satisfying

$$
\begin{array}{rlrl}
\left|G_{i}^{ \pm}(t)\right| & \leq \delta, & \left|B_{i}^{ \pm}(t)\right| \leq C, \\
\left|h_{i}^{ \pm}(t)-H(t)\right| & \leq \delta, & \left|D_{j}\right| \leq \delta, \\
|\lambda| & \leq \delta
\end{array}
$$

for some $\delta>0$ sufficiently small and a bounded function $H$ defined on $\mathbb{R}$. In Section 3.3, we substitute the expressions (3.8) into (3.9).

We concentrate on $|\lambda|<\delta$, whence $|G(t)+\lambda B(t)|<\delta$. Hence, (3.9)(i) is a small perturbation of

$$
\dot{w}^{ \pm}=D_{u} f\left(q^{ \pm}(\mu), \mu\right) w^{ \pm},
$$

which is independent of $i$ and $N$. In particular, it does not depend on the $N$-pulse $q_{N}$ under consideration. This fact is exploited next.

Lemma 3.2. The evolution $\Phi_{ \pm}(\mu ; t, s)$ of (3.11) can be decomposed into $\Phi_{ \pm}(\mu ; t, s)$ $=\Phi_{ \pm}^{s}(\mu ; t, s)+\Phi_{ \pm}^{u}(\mu ; t, s)$ defined for $s, t \geq 0$ or $s, t \leq 0$, respectively. The functions

$$
\Phi_{ \pm}^{s}(\mu ; t, t)=: P_{ \pm}^{s}(\mu)(t), \quad \Phi_{ \pm}^{u}(\mu ; t, t)=: P_{ \pm}^{u}(\mu)(t)
$$

are projections and the estimates

$$
\left.\begin{array}{l}
\left.\begin{array}{l}
\left|\Phi_{+}^{s}(\mu ; t, s)\right| \leq C e^{-\alpha^{s}(t-s)} \\
\left|\Phi_{+}^{u}(\mu ; s, t)\right| \leq C e^{-\alpha^{u}(t-s)}
\end{array}\right\}, \quad 0 \leq s \leq t, \\
\left|\Phi_{-}^{s}(\mu ; t, s)\right| \leq C e^{-\alpha^{s}(t-s)} \\
\left|\Phi_{-}^{u}(\mu ; s, t)\right| \leq C e^{-\alpha^{u}(t-s)}
\end{array}\right\}, \quad s \leq t \leq 0,
$$


hold. The ranges of the projections at $t=0$ are given by

$$
\begin{array}{ll}
R P_{+}^{s}(\mu)(0)=\mathbb{C} \dot{q}_{1}(0) \oplus Y^{+}, & R P_{+}^{u}(\mu)(0)=\mathbb{C} \psi_{1}(0) \oplus Y^{-}, \\
R P_{-}^{u}(\mu)(0)=\mathbb{C} \dot{q}_{1}(0) \oplus Y^{-}, & R P_{+}^{s}(\mu)(0)=\mathbb{C} \psi_{1}(0) \oplus Y^{+},
\end{array}
$$

In addition,

$$
\begin{aligned}
& \left|P_{+}^{k}(\mu)(t)-P_{0}^{k}\right| \leq C e^{-\alpha^{s} t}, \quad t \geq 0 \\
& \left|P_{-}^{k}(\mu)(t)-P_{0}^{k}\right| \leq C e^{\alpha^{u} t}, \quad t \leq 0,
\end{aligned}
$$

holds for $k=s, u$.

Proof. See [San93, Lemma 1.1] and Lemma 2.1. Note that equation (3.11) is defined on $\mathbb{R}^{n}$. However, the estimates carry over immediately to the complex field.

We define the spaces

$$
\begin{aligned}
V_{w} & :=\bigoplus_{i=1}^{N} C^{0}\left(\left[-T_{i-1}, 0\right], \mathbb{C}^{n}\right) \oplus C^{0}\left(\left[0, T_{i}\right], \mathbb{C}^{n}\right), \\
V_{a} & :=\bigoplus_{j=1}^{N-1} E^{s} \oplus E^{u}, \\
V_{b} & :=\bigoplus_{i=1}^{N} R P_{-}^{u}(\mu)(0) \oplus R P_{+}^{s}(\mu)(0)=\bigoplus_{i=1}^{N}\left(\mathbb{C} \dot{q}_{1}(0) \oplus Y^{-}\right) \oplus\left(\mathbb{C} \dot{q}_{1}(0) \oplus Y^{+}\right), \\
V_{\lambda} & :=U_{\delta}(0) \subset \mathbb{C}
\end{aligned}
$$

considered over the complex field. The product spaces are endowed with the maximum norm. We consider elements

$$
\begin{aligned}
& w=\left(w_{i}^{-}, w_{i}^{+}\right) \in V_{w}, \quad a=\left(a_{j}^{-}, a_{j}^{+}\right) \in V_{a}, \\
& b=\left(b_{i}^{-}, b_{i}^{+}\right) \in V_{b}, \quad \lambda \in V_{\lambda},
\end{aligned}
$$

where $i=1, \ldots, N$ and $j=1, \ldots, N-1$. We set $a_{0}^{-}=a_{N}^{+}=0$ in the following.

In order to solve (3.9), we first consider the ordinary differential equation (3.9)(i)

$$
\dot{w}_{i}^{ \pm}=D_{u} f\left(q^{ \pm}(\mu)(t), \mu\right) w_{i}^{ \pm}+\left(G_{i}^{ \pm}(t)+\lambda B_{i}^{ \pm}(t)\right) w_{i}^{ \pm}+\lambda h_{i}^{ \pm}(t) d_{i}
$$

for $i=1, \ldots, N$ on the intervals $\left(-T_{i-1}, 0\right)$ and $\left(0, T_{i}\right)$, respectively. The general solution bounded uniformly in $\left(T_{j}\right)$ solves the fixed point equation

$$
\begin{aligned}
w_{i}^{-}(t)=\Phi_{-}^{s} & \left(t,-T_{i-1}\right) a_{i-1}^{-}+\Phi_{-}^{u}(t, 0) b_{i}^{-} \\
& +\int_{0}^{t} \Phi_{-}^{u}(t, s)\left(\left(G_{i}^{-}(s)+\lambda B_{i}^{-}(s)\right) w_{i}^{-}(s)+\lambda h_{i}^{-}(s) d_{i}\right) d s \\
& +\int_{-T_{i-1}}^{t} \Phi_{-}^{s}(t, s)\left(\left(G_{i}^{-}(s)+\lambda B_{i}^{-}(s)\right) w_{i}^{-}(s)+\lambda h_{i}^{-}(s) d_{i}\right) d s, \\
w_{i}^{+}(t)=\Phi_{+}^{u}\left(t, T_{i}\right) a_{i}^{+}+\Phi_{+}^{s}(t, 0) b_{i}^{+} & \\
& +\int_{0}^{t} \Phi_{+}^{s}(t, s)\left(\left(G_{i}^{+}(s)+\lambda B_{i}^{+}(s)\right) w_{i}^{+}(s)+\lambda h_{i}^{+}(s) d_{i}\right) d s \\
& +\int_{T_{i}}^{t} \Phi_{+}^{u}(t, s)\left(\left(G_{i}^{+}(s)+\lambda B_{i}^{+}(s)\right) w_{i}^{+}(s)+\lambda h_{i}^{+}(s) d_{i}\right) d s
\end{aligned}
$$




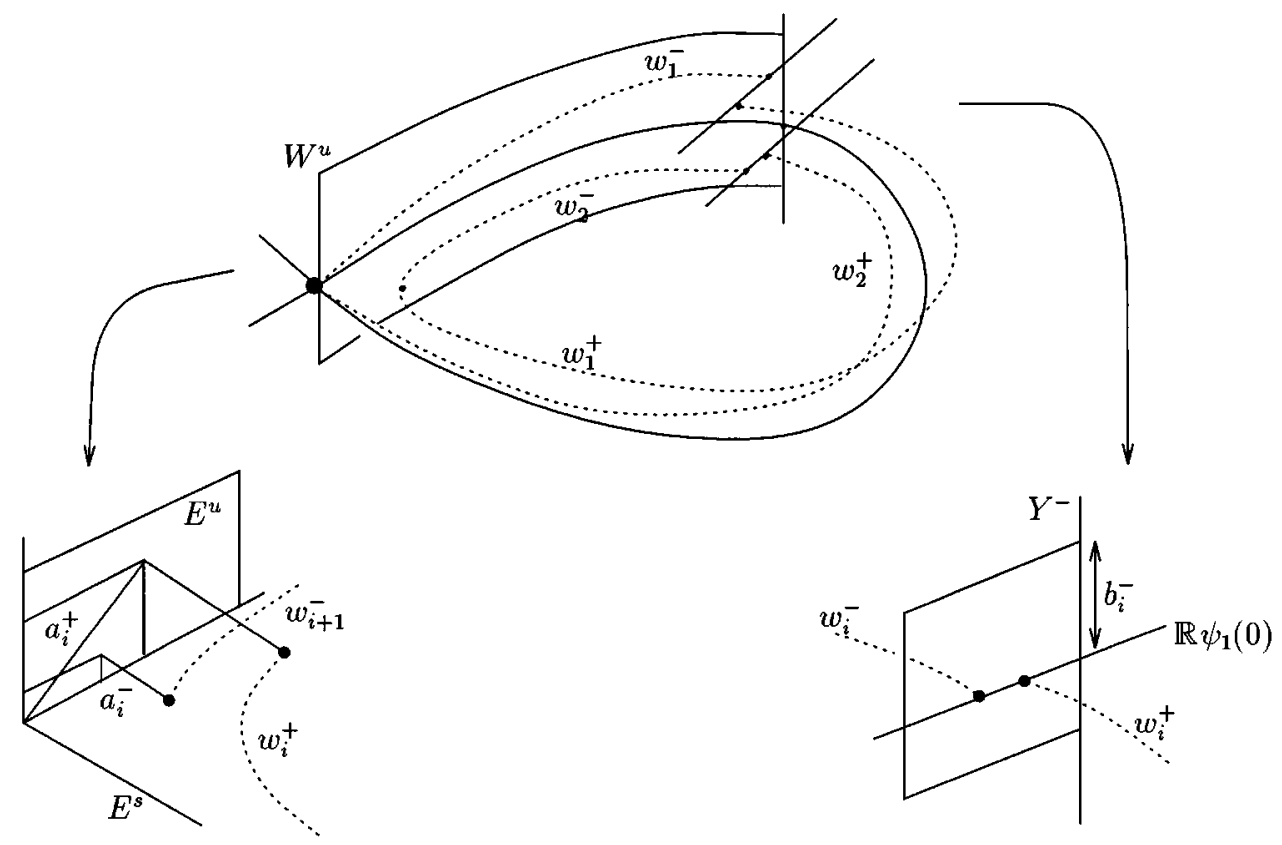

Figure 2. The interpretation of eigenfunctions as $N$-homoclinic solutions

for $i=1, \ldots, N$ and arbitrary elements $a \in V_{a}, b \in V_{b}$ and $d \in \mathbb{C}^{N}$. Here and in the sequel, we will suppress the dependence of the evolution on $\mu$. Note that the integrals in (3.14) exist even for $i=1, N$ for which $T_{0}=T_{N}=\infty$ due to the exponential decay of the evolution and the definition $a_{0}^{-}=a_{N}^{+}=0$. Indeed, the corresponding equations are given by

$$
\begin{gathered}
w_{1}^{-}(t)=\Phi_{-}^{u}(t, 0) b_{1}^{-}+\int_{0}^{t} \Phi_{-}^{u}(t, s)\left(\left(G_{1}^{-}(s)+\lambda B_{1}^{-}(s)\right) w_{1}^{-}(s)+\lambda h_{1}^{-}(s) d_{1}\right) d s \\
+\int_{-\infty}^{t} \Phi_{-}^{s}(t, s)\left(\left(G_{1}^{-}(s)+\lambda B_{1}^{-}(s)\right) w_{1}^{-}(s)+\lambda h_{1}^{-}(s) d_{1}\right) d s \\
w_{N}^{+}(t)=\Phi_{+}^{s}(t, 0) b_{N}^{+}+\int_{0}^{t} \Phi_{+}^{s}(t, s)\left(\left(G_{N}^{+}(s)+\lambda B_{N}^{+}(s)\right) w_{N}^{+}(s)+\lambda h_{N}^{+}(s) d_{N}\right) d s \\
+\int_{\infty}^{t} \Phi_{+}^{u}(t, s)\left(\left(G_{N}^{+}(s)+\lambda B_{N}^{+}(s)\right) w_{N}^{+}(s)+\lambda h_{N}^{+}(s) d_{N}\right) d s .
\end{gathered}
$$

See Figure 2 for a geometric interpretation. Instead of inverting the system (3.9) at once, we are going to invert it step by step in a sequence of lemmata for clarity.

Lemma 3.3. There exists an operator $W_{1}: V_{\lambda} \times V_{a} \times V_{b} \times \mathbb{C}^{N} \rightarrow V_{w}$ such that

$$
w:=W_{1}(\lambda)(a, b, d)
$$

solves (3.14) for any $(a, b, d)$ and $\lambda$. Moreover, any bounded solution of (3.14) is given by (3.15). The operator $W_{1}$ is analytic in $\lambda$, linear in $(a, b, d)$ and admits the 
estimate

$$
\left\|W_{1}(\lambda)(a, b, d)\right\| \leq C(|a|+|b|+|\lambda||d|)
$$

uniformly in $\left(T_{j}\right)$.

Proof. We rewrite (3.14) as

$$
\left(i d-L_{1}(\lambda)\right) w=L_{2}(\lambda)(a, b, d),
$$

where the linear operators $L_{1}(\lambda)$ and $L_{2}(\lambda)$ are given by

$$
\begin{aligned}
\left(L_{1}(\lambda) w\right)_{i}^{-}(t)= & \int_{0}^{t} \Phi_{-}^{u}(t, s)\left(G_{i}^{-}(s)+\lambda B_{i}^{-}(s)\right) w_{i}^{-}(s) d s \\
& +\int_{-T_{i-1}}^{t} \Phi_{-}^{s}(t, s)\left(G_{i}^{-}(s)+\lambda B_{i}^{-}(s)\right) w_{i}^{-}(s) d s, \\
\left(L_{1}(\lambda) w\right)_{i}^{+}(t)= & \int_{0}^{t} \Phi_{+}^{s}(t, s)\left(G_{i}^{+}(s)+\lambda B_{i}^{+}(s)\right) w_{i}^{+}(s) d s \\
& +\int_{T_{i}}^{t} \Phi_{+}^{u}(t, s)\left(G_{i}^{+}(s)+\lambda B_{i}^{+}(s)\right) w_{i}^{+}(s) d s
\end{aligned}
$$

and

$$
\begin{aligned}
\left(L_{2}(\lambda)(a, b, d)\right)_{i}^{-}(t)= & \Phi_{-}^{s}\left(t,-T_{i-1}\right) a_{i-1}^{-}+\Phi_{-}^{u}(t, 0) b_{i}^{-} \\
& +\left(\int_{0}^{t} \Phi_{-}^{u}(t, s) h_{i}^{-}(s) d s+\int_{-T_{i-1}}^{t} \Phi_{-}^{s}(t, s) h_{i}^{-}(s) d s\right) \lambda d_{i}, \\
\left(L_{2}(\lambda)(a, b, d)\right)_{i}^{+}(t)=\Phi_{+}^{u}\left(t, T_{i}\right) a_{i}^{+}+\Phi_{+}^{s}(t, 0) b_{i}^{+} & \\
& +\left(\int_{0}^{t} \Phi_{+}^{s}(t, s) h_{i}^{+}(s) d s+\int_{T_{i}}^{t} \Phi_{+}^{u}(t, s) h_{i}^{+}(s) d s\right) \lambda d_{i} .
\end{aligned}
$$

Both operators are analytic in $\lambda$ and bounded uniformly in $\left(T_{j}\right)$ considered as operators with range in $V_{w}$. In fact, the estimates

$$
\begin{aligned}
\left\|L_{1}(\lambda) w\right\| & \leq C(|G|+|\lambda|)\|w\| \leq C \delta\|w\|, \\
\left\|L_{2}(\lambda)(a, b, d)\right\| & \leq C(|a|+|b|+|\lambda||d|)
\end{aligned}
$$

hold owing to (3.10) and Lemma 3.2. Thus, the operator $i d-L_{1}(\lambda)$ is invertible on $V_{w}$. The inverse $\left(i d-L_{1}(\lambda)\right)^{-1}$ is analytic in $\lambda$ and we obtain the solution of (3.9)(i)

$$
w=\left(i d-L_{1}(\lambda)\right)^{-1} L_{2}(\lambda)(a, b, d)=: W_{1}(\lambda)(a, b, d)
$$

depending linearly on $(a, b, d) \in V_{a} \times V_{b} \times V_{d}$ and analytically on $\lambda \in V_{\lambda}$. The estimate

$$
\left\|W_{1}(\lambda)(a, b, d)\right\| \leq C(|a|+|b|+|\lambda||d|)
$$

follows from (3.20).

In the next step, we solve equation (3.9)(iv)

$$
w_{j}^{+}\left(T_{j}\right)-w_{j+1}^{-}\left(-T_{j}\right)=D_{j} d
$$

for $j=1, \ldots, N-1$. 
Lemma 3.4. There exist operators

$$
\begin{gathered}
A_{1}: V_{\lambda} \times V_{b} \times \mathbb{C}^{N} \rightarrow V_{a}, \\
W_{2}: V_{\lambda} \times V_{b} \times \mathbb{C}^{N} \rightarrow V_{w},
\end{gathered}
$$

such that

$$
(a, w):=\left(A_{1}(\lambda)(b, d), W_{2}(\lambda)(b, d)\right)
$$

solves (3.14) and (3.22) for any $(b, d)$ and $\lambda$. In addition, any bounded solution of these equations is given by (3.23). The operators $A_{1}$ and $W_{2}$ are analytic in $\lambda$, linear in $(b, d)$ and fulfill

$$
\begin{aligned}
\left|A_{1}(\lambda)(b, d)\right| & \leq C\left(\left(e^{-\alpha T}+|G|+|\lambda|\right)|b|+(|D|+|\lambda|)|d|\right), \\
\left\|W_{2}(\lambda)(b, d)\right\| & \leq C(|b|+(|D|+|\lambda|)|d|)
\end{aligned}
$$

uniformly in $\left(T_{j}\right)$. Moreover, we have

$$
\begin{aligned}
& \left(A_{1}(\lambda)(b, d)\right)_{j}^{-}=-P_{0}^{s} D_{j} d+\left(A_{2}(\lambda)(b, d)\right)_{j}^{-}, \\
& \left(A_{1}(\lambda)(b, d)\right)_{j}^{+}=P_{0}^{u} D_{j} d+\left(A_{2}(\lambda)(b, d)\right)_{j}^{+},
\end{aligned}
$$

for a bounded operator $A_{2}$ admitting the estimate

$$
\left|A_{2}(\lambda)(b, d)\right| \leq C\left(\left(e^{-\alpha T}+|G|+|\lambda|\right)|b|+((p(T)+|G|)|D|+|\lambda|)|d|\right)
$$

with $p(T):=\sup _{t \geq T}\left(\left|P_{+}^{u}(t)-P_{0}^{u}\right|+\left|P_{-}^{s}(-t)-P_{0}^{s}\right|\right)$.

Proof. Using (3.14) and (3.13) we obtain

$$
\begin{aligned}
D_{j} d=a_{j}^{+} & -a_{j}^{-}+\left(P_{+}^{u}\left(T_{j}\right)-P_{0}^{u}\right) a_{j}^{+}+\left(P_{0}^{s}-P_{-}^{s}\left(-T_{j}\right)\right) a_{j}^{-} \\
& +\Phi_{+}^{s}\left(T_{j}, 0\right) b_{j}^{+}-\Phi_{-}^{u}\left(-T_{j}, 0\right) b_{j+1}^{-} \\
& +\int_{0}^{T_{j}} \Phi_{+}^{s}\left(T_{j}, s\right)\left(\left(G_{j}^{+}(s)+\lambda B_{j}^{+}(s)\right) w_{j}^{+}(s)+\lambda h_{j}^{+}(s) d_{j}\right) d s \\
& +\int_{-T_{j}}^{0} \Phi_{-}^{u}\left(-T_{j}, s\right)\left(\left(G_{j+1}^{-}(s)+\lambda B_{j+1}^{-}(s)\right) w_{j+1}^{-}(s)+\lambda h_{j+1}^{-}(s) d_{j+1}\right) d s
\end{aligned}
$$

for $j=1, \ldots, N-1$. Here, $P_{+}^{s}(t)$ and $P_{-}^{u}(t)$ are defined in Lemma 3.2. We substitute $w=W_{1}(\lambda)(a, b, d)$ from Lemma 3.3 into (3.27). Then (3.27) defines an equation

$$
D_{j} d=a_{j}^{+}-a_{j}^{-}+\left(L_{3}(\lambda)(a, b, d)\right)_{j}
$$

linear in $(a, b, d)$ and analytic in $\lambda$. Owing to the estimates in Lemma 3.2 and (3.18), (3.16) we obtain

$$
\begin{aligned}
\left|L_{3}(\lambda)(a, b, d)\right| & \leq C\left(p(T)|a|+e^{-\alpha T}|b|+(|G|+|\lambda|)|| W_{1}(\lambda)(a, b, d) \|+|\lambda||d|\right) \\
& \leq C\left((p(T)+|G|+|\lambda|)|a|+\left(e^{-\alpha T}+|G|+|\lambda|\right)|b|+|\lambda||d|\right) \\
& \leq C \delta(|a|+|b|+|d|)
\end{aligned}
$$


for $T$ large, where $p(T)$ was defined in the statement of the lemma. The map $J_{1}$ defined by

$$
\begin{aligned}
V_{a} & \rightarrow \bigoplus_{j=1}^{N-1} \mathbb{C}^{n}, \\
\left(a_{j}^{+}, a_{j}^{-}\right) & \mapsto\left(a_{j}^{+}-a_{j}^{-}\right)
\end{aligned}
$$

is a linear isomorphism due to $E^{u} \times E^{s}=\mathbb{C}^{n}$. For $\delta>0$ sufficiently small, we can therefore invert the operator

$$
a \mapsto J_{1} a+L_{3}(\lambda)(a, 0,0),
$$

yielding the solution operator

$$
a=\left(J_{1}+L_{3}(\lambda) I_{1}\right)^{-1}\left(D d-L_{3}(\lambda)(0, b, d)\right)=: A_{1}(\lambda)(b, d)
$$

of (3.28) using the definition $I_{1} a=(a, 0,0)$. Moreover, we define

$$
W_{2}(\lambda)(b, d):=W_{1}(\lambda)\left(A_{1}(\lambda)(b, d), b, d\right) .
$$

Then

$$
(\lambda, a, b, d, w)=\left(\lambda, A_{1}(\lambda)(b, d), b, d, W_{2}(\lambda)(b, d)\right)
$$

solves (3.14) and (3.22) by Lemma 3.3 and the statements above. From (3.29) we conclude

$$
\left|A_{1}(\lambda)(b, d)\right| \leq C\left(\left(e^{-\alpha T}+|G|+|\lambda|\right)|b|+(|D|+|\lambda|)|d|\right),
$$

which proves the first part of (3.24). Using this estimate together with (3.16) and the definition of $W_{2}$ yields the second part of (3.24), namely

$$
\left\|W_{2}(\lambda)(a, b, d)\right\| \leq C(|b|+(|D|+\lambda \mid)|d|) .
$$

It remains to prove the decomposition (3.25). Projecting (3.28) into $E^{s}$ and $E^{u}$ by $P_{0}^{s}$ and $P_{0}^{u}$, respectively, yields

$$
\begin{aligned}
& a_{j}^{-}=-P_{0}^{s} D_{j} d+P_{0}^{s}\left(L_{3}(\lambda)(a, b, d)\right)_{j}, \\
& a_{j}^{+}=P_{0}^{u} D_{j} d-P_{0}^{u}\left(L_{3}(\lambda)(a, b, d)\right)_{j} .
\end{aligned}
$$

Substituting $a=A_{1}(\lambda)(b, d)$ into (3.31) gives

$$
\begin{aligned}
\left(A_{1}(\lambda)(b, d)\right)_{j}^{-} & =-P_{0}^{s} D_{j} d+P_{0}^{s}\left(L_{3}(\lambda)\left(A_{1}(\lambda)(b, d), b, d\right)\right)_{j} \\
& =:-P_{0}^{s} D_{j} d+\left(A_{2}(\lambda)(b, d)\right)_{j}^{-} \\
\left(A_{1}(\lambda)(b, d)\right)_{j}^{+} & =P_{0}^{u} D_{j} d-P_{0}^{u}\left(L_{3}(\lambda)\left(A_{1}(\lambda)(b, d), b, d\right)\right)_{j} \\
& =: P_{0}^{u} D_{j} d+\left(A_{2}(\lambda)(b, d)\right)_{j}^{+} .
\end{aligned}
$$

Using the estimates (3.29) for $L_{3}$ and (3.24) for $A_{1}$ finally gives (3.26). This proves the lemma.

We have to investigate the remaining equations (3.9)(ii) and (iii)

$$
\begin{aligned}
& w_{i}^{-}(0) \in \mathbb{C} \psi_{1}(0) \oplus Y^{+} \oplus Y^{-}, \\
& w_{i}^{+}(0) \in \mathbb{C} \psi_{1}(0) \oplus Y^{+} \oplus Y^{-}, \\
& w_{i}^{+}(0)-w_{i}^{-}(0) \in \mathbb{C} \psi_{1}(0),
\end{aligned}
$$


for $i=1, \ldots, N$, where we substitute $w=W_{2}(\lambda)(b, d)$ defined in Lemma 3.4. Owing to $(3.14)$, we have

$$
\begin{aligned}
w_{i}^{-}(0)= & \Phi_{-}^{s}\left(0,-T_{i-1}\right) a_{i-1}^{-}+b_{i}^{-} \\
& \quad+\int_{-T_{i-1}}^{0} \Phi_{-}^{s}(0, s)\left(\left(G_{i}^{-}(s)+\lambda B_{i}^{-}(s)\right) w_{i}^{-}(s)+\lambda h_{i}^{-}(s) d_{i}\right) d s, \\
w_{i}^{+}(0)= & \Phi_{+}^{u}\left(0, T_{i}\right) a_{i}^{+}+b_{i}^{+} \\
& \quad+\int_{T_{i}}^{0} \Phi_{+}^{u}(0, s)\left(\left(G_{i}^{+}(s)+\lambda B_{i}^{+}(s)\right) w_{i}^{+}(s)+\lambda h_{i}^{+}(s) d_{i}\right) d s,
\end{aligned}
$$

with $w$ and $a$ given by (3.23). By definition (3.12) and (3.13), we can decompose $b$ in a unique way according to

$$
b_{i}^{-}=x_{i}^{-}+y_{i}^{-}, \quad b_{i}^{+}=x_{i}^{+}+y_{i}^{+},
$$

such that $x_{i}^{ \pm} \in \mathbb{C} \dot{q}_{1}(0)$ and $y_{i}^{ \pm} \in Y^{ \pm}$, respectively. In other words,

$$
\begin{aligned}
& b=x+y, \\
& x \in \bigoplus_{i=1}^{N} \mathbb{C} \dot{q}_{1}(0) \times \mathbb{C} \dot{q}_{1}(0), \\
& y \in \bigoplus_{i=1}^{N} Y^{-} \times Y^{+} .
\end{aligned}
$$

Next we use the fact (2.5), namely that

$$
\mathbb{C}^{n}=\mathbb{C} \psi_{1}(0) \oplus \mathbb{C} \dot{q}_{1}(0) \oplus Y^{+} \oplus Y^{-} .
$$

Then (3.32) is equivalent to the system of equations

$$
\begin{array}{r}
P\left(\mathbb{C} \dot{q}_{1}(0), \mathbb{C} \psi_{1}(0) \oplus Y^{+} \oplus Y^{-}\right) w_{i}^{-}(0)=0, \\
P\left(\mathbb{C} \dot{q}_{1}(0), \mathbb{C} \psi_{1}(0) \oplus Y^{+} \oplus Y^{-}\right) w_{i}^{+}(0)=0, \\
P\left(Y^{+} \oplus Y^{-}, \mathbb{C} \dot{q}_{1}(0) \oplus \mathbb{C} \psi_{1}(0)\right)\left(w_{i}^{+}(0)-w_{i}^{-}(0)\right)=0,
\end{array}
$$

where $P(X, Y)$ denotes the projection onto $X$ with kernel $Y$. Indeed, note that the components of $w_{i}^{-}(0)$ and $w_{i}^{+}(0)$ in $\mathbb{C} \dot{q}_{1}(0)$ vanish due to the first two equations, whence it is sufficient to project onto $Y^{+} \oplus Y^{-}$in the third equation.

Lemma 3.5. There exist operators

$$
\begin{aligned}
& B_{1}: V_{\lambda} \times \mathbb{C}^{N} \rightarrow V_{b}, \\
& A_{3}: V_{\lambda} \times \mathbb{C}^{N} \rightarrow V_{a}, \\
& W_{3}: V_{\lambda} \times \mathbb{C}^{N} \rightarrow V_{w},
\end{aligned}
$$

such that

$$
(a, b, w):=\left(A_{3}(\lambda) d, B_{1}(\lambda) d, W_{3}(\lambda) d\right)
$$

solves (3.14), (3.22) and (3.32) for any $d$ and $\lambda$. Any bounded solution of these equations is given by (3.36). The operators $A_{3}, B_{1}$ and $W_{3}$ are analytic in $\lambda$ and 
linear in $d$. They satisfy

$$
\begin{aligned}
\left|A_{3}(\lambda) d\right| & \leq C(|D|+|\lambda|)|d| \\
\left|B_{1}(\lambda) d\right| & \leq C\left(\left(e^{-\alpha T}+|G|\right)|D|+|\lambda|\right)|d|, \\
\left\|W_{3}(\lambda) d\right\| & \leq C(|D|+|\lambda|)|d|
\end{aligned}
$$

uniformly in $\left(T_{j}\right)$. Again $A_{3}(\lambda)$ can be written according to

$$
\begin{aligned}
& \left(A_{3}(\lambda) d\right)_{j}^{-}=-P_{0}^{s} D_{j} d+\left(A_{4}(\lambda) d\right)_{j}^{-}, \\
& \left(A_{3}(\lambda) d\right)_{j}^{+}=P_{0}^{u} D_{j} d+\left(A_{4}(\lambda) d\right)_{j}^{+},
\end{aligned}
$$

for some operator $A_{4}$ linear in $d$ and analytic in $\lambda$ such that

$$
\left|A_{4}(\lambda) d\right| \leq C\left(\left(e^{-\alpha T}+|G|\right)|D|+|\lambda|\right)|d|
$$

holds.

Proof. First we substitute (3.33) into (3.35). Using (3.34) we obtain therefore the equations

$$
\begin{aligned}
& 0=x_{i}^{-}+ P\left(\mathbb{C} \dot{q}_{1}(0), \mathbb{C} \psi_{1}(0) \oplus Y^{+} \oplus Y^{-}\right)\left(\Phi_{-}^{s}\left(0,-T_{i-1}\right) a_{i-1}^{-}\right. \\
&\left.\quad \int_{-T_{i-1}}^{0} \Phi_{-}^{s}(0, s)\left(\left(G_{i}^{-}(s)+\lambda B_{i}^{-}(s)\right) w_{i}^{-}(s)+\lambda h_{i}^{-}(s) d_{i}\right) d s\right) \\
& 0=x_{i}^{+}+ P\left(\mathbb{C} \dot{q}_{1}(0), \mathbb{C} \psi_{1}(0) \oplus Y^{+} \oplus Y^{-}\right)\left(\Phi_{+}^{u}\left(0, T_{i}\right) a_{i}^{+}\right. \\
&\left.+\int_{T_{i}}^{0} \Phi_{+}^{u}(0, s)\left(\left(G_{i}^{+}(s)+\lambda B_{i}^{+}(s)\right) w_{i}^{+}(s)+\lambda h_{i}^{+}(s) d_{i}\right) d s\right) \\
& 0=y_{i}^{+}-y_{i}^{-} \\
&+ P\left(Y^{+} \oplus Y^{-}, \mathbb{C} \dot{q}_{1}(0) \times \mathbb{C} \psi_{1}(0)\right)\left(\Phi_{+}^{u}\left(0, T_{i}\right) a_{i}^{+}-\Phi_{-}^{s}\left(0,-T_{i-1}\right) a_{i-1}^{-}\right. \\
&+ \int_{T_{i}}^{0} \Phi_{+}^{u}(0, s)\left(\left(G_{i}^{+}(s)+\lambda B_{i}^{+}(s)\right) w_{i}^{+}(s)+\lambda h_{i}^{+}(s) d_{i}\right) d s \\
&\left.\quad-\int_{-T_{i-1}}^{0} \Phi_{-}^{s}(0, s)\left(\left(G_{i}^{-}(s)+\lambda B_{i}^{-}(s)\right) w_{i}^{-}(s)+\lambda h_{i}^{-}(s) d_{i}\right) d s\right)
\end{aligned}
$$

They can be written in the form

$$
\left(\begin{array}{c}
x_{i}^{-} \\
x_{i}^{+} \\
y_{i}^{+}-y_{i}^{-}
\end{array}\right)+\left(L_{4}(\lambda)(b, d)\right)_{i}=0
$$


by substituting (3.23). Owing to Lemma 3.2 and (3.24), we obtain the estimate

$$
\begin{aligned}
\left|L_{4}(\lambda)(b, d)\right| \leq & C\left(e^{-\alpha T}|a|+(|G|+|\lambda|)|w|+|\lambda||d|\right) \\
\leq & C\left(e^{-\alpha T}\left(\left(e^{-\alpha T}+|G|+|\lambda|\right)|b|+(|D|+|\lambda|)|d|\right)\right. \\
& +(|G|+|\lambda|)(|b|+(|D|+|\lambda|)|d|)+|\lambda||d|) \\
\leq & C\left(\left(e^{-2 \alpha T}+|G|+|\lambda|\right)|b|+\left(\left(e^{-\alpha T}+|G|\right)|D|+|\lambda|\right)|d|\right) \\
\leq & C\left(\left(e^{-2 \alpha T}+|G|+|\lambda|\right)(|x|+|y|)+\left(\left(e^{-\alpha T}+|G|\right)|D|+|\lambda|\right)|d|\right) \\
\leq & C \delta(|x|+|y|)+C\left(\left(e^{-\alpha T}+|G|\right)|D|+|\lambda|\right)|d| .
\end{aligned}
$$

Note that $b=x+y$ by (3.34). Moreover, the map $J_{2}$ defined by

$$
\begin{gathered}
\left(\bigoplus_{i=1}^{N} \mathbb{C} \dot{q}_{1}(0) \oplus \mathbb{C} \dot{q}_{1}(0)\right) \oplus\left(\bigoplus_{i=1}^{N} Y^{-} \oplus Y^{+}\right) \rightarrow \bigoplus_{i=1}^{N} \mathbb{C} \dot{q}_{1}(0) \oplus \mathbb{C} \dot{q}_{1}(0) \oplus\left(Y^{-} \oplus Y^{+}\right), \\
\left(\left(x_{i}^{-}, x_{i}^{+}\right),\left(y_{i}^{-}, y_{i}^{+}\right)\right) \mapsto\left(x_{i}^{-}, x_{i}^{+}, y_{i}^{+}-y_{i}^{-}\right)
\end{gathered}
$$

is an isomorphism due to (2.5). Thus, the operator

$$
(x, y) \mapsto J_{2}(x+y)+L_{4}(\lambda)(x+y, 0)
$$

is invertible due to (3.42), whence

$$
b=-\left(J_{2}+L_{4}(\lambda) I_{2}\right)^{-1} L_{4}(\lambda)(0, d)=: B_{1}(\lambda) d
$$

solves (3.41). Here, we used the definition $I_{2} b=(b, 0)$. From (3.42) we conclude the estimate

$$
\left|B_{1}(\lambda) d\right| \leq C\left(\left(e^{-\alpha T}+|G|\right)|D|+|\lambda|\right)|d| .
$$

Substituting $b=B_{1}(\lambda) d$ into the operators $A_{1}$ and $W_{2}$ given in (3.23) defines $A_{3}$ and $W_{3}$. The estimate (3.37) follows now easily from (3.44) and (3.24). In the same way, one obtains

$$
A_{4}(\lambda) d=A_{2}(\lambda)\left(B_{1}(\lambda) d, d\right)
$$

using (3.25) and the estimate (3.39) by using (3.26). The lemma is proved.

Let us summarize the results obtained so far. By Lemmata 3.3, 3.4 and 3.5 we have shown that $(\lambda, a, b, d, w)$ is a bounded solution of (3.9) if and only if it is given by

$$
(\lambda, a, b, d, w):=\left(\lambda, A_{3}(\lambda) d, B_{1}(\lambda) d, d, W_{3}(\lambda) d\right) .
$$

With $(\lambda, a, b, d, w)$ at hand, the equations

$$
w_{i}^{+}(0)=w_{i}^{-}(0), \quad i=1, \ldots, N,
$$

are fulfilled if and only if the jumps

$$
\xi_{i}=\left\langle\psi_{1}(0), w_{i}^{+}(0)-w_{i}^{-}(0)\right\rangle
$$

vanish identically. Indeed, then $w_{i}^{+}(0)=w_{i}^{-}(0)$ holds due to (3.9)(iii). In the next step, we derive formulae for the jumps $\xi_{i}$. 
Lemma 3.6. Let $(\lambda, a, b, d, w)$ be given by (3.45). Then the relation $w_{i}^{+}(0)=$ $w_{i}^{-}(0)$ holds for $i=1, \ldots, N$ if and only if

$$
\xi_{i}=\left\langle\psi_{1}(0), w_{i}^{+}(0)-w_{i}^{-}(0)\right\rangle=0 .
$$

Moreover, the jumps $\xi_{i}$ can be written as

$$
\begin{array}{r}
\xi_{i}=\left\langle\psi\left(T_{i}\right), P_{0}^{u} D_{i} d\right\rangle+\left\langle\psi\left(-T_{i-1}\right), P_{0}^{s} D_{i-1} d\right\rangle \\
-\lambda \int_{-\infty}^{\infty}\langle\psi(t), H(t)\rangle d t d_{i}+(R(\lambda) d)_{i}
\end{array}
$$

for some function $R: V_{\lambda} \times \mathbb{C}^{N} \rightarrow \mathbb{C}^{N}$ analytic in $\lambda$ and linear in $d$ admitting the estimate

$$
|R(\lambda) d| \leq C\left(\left(e^{-\alpha T}+|G|\right)^{2}|D|+\left(e^{-\alpha T}+|G|+\left\|h_{i}-H\right\|+|\lambda|\right)|\lambda|\right)|d| .
$$

Proof. Throughout, we choose $(\lambda, a, b, d, w)$ according to (3.45). Hence, $\xi_{i}$ is linear in $d$ and analytic in $\lambda$. Owing to Lemma 2.2 and (3.33) we obtain

$$
\begin{aligned}
\left\langle\psi_{1}(0), w_{i}^{+}(0)-w_{i}^{-}(0)\right\rangle=\left\langle\psi(\mu)(0), w_{i}^{+}(0)-w_{i}^{-}(0)\right\rangle & \\
= & \left\langle(\mu)(0), \Phi_{+}^{u}\left(0, T_{i}\right) a_{i}^{+}-\Phi_{-}^{s}\left(0,-T_{i-1}\right) a_{i-1}^{-}\right. \\
& \quad+\int_{T_{i}}^{0} \Phi_{+}^{u}(0, s)\left(\left(G_{i}^{+}(s)+\lambda B_{i}^{+}(s)\right) w_{i}^{+}(s)+\lambda h_{i}^{+}(s) d_{i}\right) d s \\
& \left.-\int_{-T_{i-1}}^{0} \Phi_{-}^{s}(0, s)\left(\left(G_{i}^{-}(s)+\lambda B_{i}^{-}(s)\right) w_{i}^{-}(s)+\lambda h_{i}^{-}(s) d_{i}\right) d s\right\rangle \\
=\langle & \left.\left(T_{i}\right), a_{i}^{+}\right\rangle-\left\langle\psi\left(-T_{i-1}\right), a_{i-1}^{-}\right\rangle \\
& -\int_{0}^{T_{i}}\left\langle\psi(t),\left(G_{i}^{+}(t)+\lambda B_{i}^{+}(t)\right) w_{i}^{+}(t)+\lambda h_{i}^{+}(t) d_{i}\right\rangle d t \\
& -\int_{-T_{i-1}}^{0}\left\langle\psi(t),\left(G_{i}^{-}(t)+\lambda B_{i}^{-}(t)\right) w_{i}^{-}(t)+\lambda h_{i}^{-}(t) d_{i}\right\rangle d t
\end{aligned}
$$

by using Lemma 2.2. Next we estimate the expressions appearing in (3.48) term by term. Substituting (3.38) yields

$$
\begin{aligned}
& \left\langle\psi\left(T_{i}\right), a_{i}^{+}\right\rangle-\left\langle\psi\left(-T_{i-1}\right), a_{i-1}^{-}\right\rangle \\
& \quad=\left\langle\psi\left(T_{i}\right), P_{0}^{u} D_{i} d\right\rangle+\left\langle\psi\left(-T_{i-1}\right), P_{0}^{s} D_{i} d\right\rangle+O\left(e^{-\alpha T}\left(\left(e^{-\alpha T}+|G|\right)|D|+|\lambda|\right)|d|\right)
\end{aligned}
$$

using the estimate (3.39) and

$$
|\psi(t)| \leq C e^{-\alpha|t|}
$$

Owing to (3.50) we also obtain

$$
\begin{aligned}
\int_{0}^{T_{i}}\left\langle\psi(t), h_{i}^{+}(t) d_{i}\right\rangle d t+\int_{-T_{i-1}}^{0}\left\langle\psi(t), h_{i}^{-}(t) d_{i}\right\rangle d t \\
\quad=\int_{-\infty}^{\infty}\langle\psi(t), H(t)\rangle d t+O\left(e^{-\alpha T}\|H\|+\left\|h_{i}-H\right\|\right) .
\end{aligned}
$$


Substituting (3.37) obtains

$$
\begin{gathered}
\left|\int_{0}^{T_{i}}\left\langle\psi(t), B_{i}^{+}(t) w_{i}^{+}(t)\right\rangle d t+\int_{-T_{i-1}}^{0}\left\langle\psi(t), B_{i}^{-}(t) w_{i}^{-}(t)\right\rangle d t\right| \\
\leq C(|D|+|\lambda|)|d| .
\end{gathered}
$$

Therefore, it remains to estimate the expression

$$
\int_{0}^{T_{i}}\left\langle\psi(t), G_{i}^{+}(t) w_{i}^{+}(t)\right\rangle d t+\int_{-T_{i-1}}^{0}\left\langle\psi(t), G_{i}^{-}(t) w_{i}^{-}(t)\right\rangle d t
$$

In order to get a sharper estimate, we have to exploit the inequalities

$$
\begin{aligned}
\left|w_{i}^{-}(t)\right| & \leq C\left(e^{-\alpha^{s}\left(T_{i-1}+t\right)}\left|a_{i-1}^{-}\right|+\left|b_{i}^{-}\right|+(|G|+|\lambda|)\|w\|+|\lambda||d|\right) \\
& \leq C\left(\left(e^{-\alpha^{s}\left(T_{i-1}+t\right)}+e^{-\alpha T}+|G|\right)|D|+|\lambda|\right)|d|, \\
\left|w_{i}^{+}(t)\right| & \leq C\left(e^{-\alpha^{u}\left(T_{i}-t\right)}\left|a_{i}^{+}\right|+\left|b_{i}^{+}\right|+(|G|+|\lambda|)\|w\|+|\lambda||d|\right) \\
& \leq C\left(\left(e^{-\alpha^{u}\left(T_{i}-t\right)}+e^{-\alpha T}+|G|\right)|D|+|\lambda|\right)|d|,
\end{aligned}
$$

which follow from (3.14), (3.37) and Lemma 3.2. Substituting these estimates into (3.53) yields

$$
\begin{aligned}
\left|\int_{0}^{T_{i}}\left\langle\psi(t), G_{i}^{+}(t) w_{i}^{+}(t)\right\rangle d t\right| & \leq\left|\int_{0}^{T_{i}}\right| \psi(t)|| G|| w_{i}^{+}(t)|d t| \\
& \leq C\left|\int_{0}^{T_{i}} e^{-\alpha t}\right| G|| w_{i}^{+}(t)|d t| \\
& \leq C|G|\left(\left(e^{-\alpha T}+|G|\right)|D|+|\lambda|\right)|d|
\end{aligned}
$$

and similarly

$$
\left|\int_{-T_{i-1}}^{0}\left\langle\psi(t), G_{i}^{-}(t) w_{i}^{-}(t)\right\rangle d t\right| \leq C|G|\left(\left(e^{-\alpha T}+|G|\right)|D|+|\lambda|\right)|d| .
$$

Here, we made the constant $\alpha$ appearing in (3.50) a bit larger than $\min \left(\alpha^{s}, \alpha^{u}\right)$, which is possible due to (H1).

Summarizing the estimates (3.49), (3.51), (3.52) and (3.54), (3.55) yields

$$
\xi_{i}=\left\langle\psi\left(T_{i}\right), P_{0}^{u} D_{i} d\right\rangle+\left\langle\psi\left(-T_{i-1}\right), P_{0}^{s} D_{i-1} d\right\rangle-\lambda \int_{-\infty}^{\infty}\langle\psi(t), H(t)\rangle d t d_{i}+(R(\lambda) d)_{i}
$$

and

$$
|R(\lambda) d| \leq C\left(\left(e^{-\alpha T}+|G|\right)^{2}|D|+|\lambda|\left(e^{-\alpha T}+|G|+\left\|h_{i}-H\right\|+|\lambda|\right)\right)|d| .
$$

This proves the lemma.

3.3. The substitution. Finally, we return to the original equations (3.6). Substituting the estimates obtained in Lemma 3.1 into the inequalities appearing in Lemma 3.6 shows that

$$
\begin{aligned}
\xi_{i}=\langle & \left.\psi\left(T_{i}\right), \dot{q}^{-}\left(-T_{i}\right)\right\rangle\left(d_{i+1}-d_{i}\right)+\left\langle\psi\left(-T_{i-1}\right), \dot{q}^{+}\left(T_{i-1}\right)\right\rangle\left(d_{i}-d_{i-1}\right) \\
& -\int_{-\infty}^{\infty}\left\langle\psi_{1}(t), B\left(q_{1}(t), \mu_{1}\right) \dot{q}_{1}(t)\right\rangle d t \lambda d_{i}+(R(\lambda) d)_{i}
\end{aligned}
$$


and

$$
|R(\lambda) d| \leq C\left(e^{-2 \alpha T} \sup _{t \geq T}\left(\left|q^{+}(t)\right|+\left|q^{-}(-t)\right|\right)+|\lambda|\left(e^{-\alpha T}+\left|\mu_{N}-\mu_{1}\right|+|\lambda|\right)\right)|d| .
$$

By Lemma 3.6, (3.6) is solved iff (3.7) and $\xi=0$ are solved with the substitutions made in (3.8). Therefore, Theorem 2 is proved.

Remark. By examining (3.8) and Lemma 3.6, we observe that the reduced function $E(\lambda)$ associated with an $N$-pulse is a small perturbation of $E_{1}^{N}(\lambda)$. Here, $E_{1}(\lambda)$ denotes the reduced function associated with the primary pulse.

Finally, for future reference, let us define the solution operator

$$
v=V(\lambda) d=\mathcal{U} d+W(\lambda) d
$$

according to (3.4) and (3.5), where we substituted (3.45) for $w$.

\section{A multiplicity Lemma}

In this section we consider the steady state equation associated with (1.2), that is, equation (1.3). We are going to subsume the term $c U_{\xi}$ into either the nonlinearity or the operator $\mathcal{A}$. This is to avoid difficulties arising if the wave speed $c=0$ equals zero and the original equation (1.3) contains a coupled ODE at the same time. We are not considering this case. Therefore, consider the equation

$$
\mathcal{A} U+F(U, \mu)=0,
$$

where $\mathcal{A}$ is given by

$$
\mathcal{A} U=\left(\begin{array}{cccc}
D_{0} \frac{d}{d x} & & & \\
& D_{1} \Delta & & \\
& & \ddots & \\
& & & (-1)^{l+1} D_{l} \Delta^{l}
\end{array}\right)\left(\begin{array}{c}
U_{0} \\
U_{1} \\
\vdots \\
U_{l}
\end{array}\right) .
$$

Here, $U=\left(U_{0}, \ldots, U_{l}\right) \in B U\left(\mathbb{R}, \mathbb{R}^{k}\right)$ is uniformly continuous with components satisfying $U_{j} \in B U\left(\mathbb{R}, \mathbb{R}^{k_{j}}\right)$. The matrices $D_{j}$ are positive definite. Therefore, $\mathcal{A}$ is a linear, densely defined and closed operator on $B U\left(\mathbb{R}, \mathbb{R}^{k}\right)$. Of course, $\mathcal{A}$ need not contain all of the blocks appearing in (4.2).

Next we define the operators $\Gamma_{j}$ and $\Gamma$ by

$$
\begin{aligned}
\Gamma_{j}: B U\left(\mathbb{R}, \mathbb{R}^{k_{j}}\right) & \rightarrow B U\left(\mathbb{R}, \mathbb{R}^{n_{j}}\right), \quad \Gamma_{j} U_{j}=\left(U_{j}, \frac{d}{d x} U_{j}, \ldots, \frac{d^{2 j-1}}{d x^{2 j-1}} U_{j}\right), \\
\Gamma: B U\left(\mathbb{R}, \mathbb{R}^{k}\right) & \rightarrow B U\left(\mathbb{R}, \mathbb{R}^{n}\right), \quad \Gamma U=\left(\Gamma_{0} U_{0}, \ldots, \Gamma_{l} U_{l}\right),
\end{aligned}
$$

for $n_{j}=2 j k_{j}$ and $n=\sum_{j=0}^{l} n_{j}$. Then $\Gamma$ is densely defined and closed. Moreover, let

$$
\begin{aligned}
B_{j}: \mathbb{R}^{k_{j}} & \rightarrow \prod_{i=1}^{2 j} \mathbb{R}^{k_{j}}=\mathbb{R}^{n_{j}}, & B_{j} U_{j} & =\left(0, \ldots, 0, U_{j}\right), \\
B: \mathbb{R}^{k} & \rightarrow \mathbb{R}^{n}, & B U & =\left(B_{0} U_{0}, \ldots, B_{l} U_{l}\right) .
\end{aligned}
$$


Now let $u=\left(u_{0}, \ldots, u_{l}\right) \in \mathbb{R}^{n}$ such that $u_{j} \in \mathbb{R}^{n_{j}}$ for $j=0, \ldots, l$. We extend the matrices $B_{j}$ and $B$ to $\mathbb{R}^{n_{j}}$ and $\mathbb{R}^{n}$, respectively, by projecting

$$
\mathbb{R}^{n_{j}}=\prod_{i=1}^{2 j} \mathbb{R}^{k_{j}} \rightarrow \mathbb{R}^{k_{j}}
$$

onto the first component of the product along the others. Finally define the Jordanblock matrices

$$
\begin{gathered}
C_{j}: \mathbb{R}^{n_{j}} \rightarrow \mathbb{R}^{n_{j}}, \quad\left(C_{j} u_{j}\right)_{i}:=u_{j, i+1}, i=1, \ldots, n_{j}-1, \\
\left(C_{j} u_{j}\right)_{n_{j}}=0
\end{gathered}
$$

and let $C u=\left(C_{0} u_{0}, \ldots, C_{l} u_{l}\right)$.

The nonlinearity $F(U, \mu)$ is a Nemitskii operator defined by

$$
F(U, \mu)(x)=G\left(\left(\frac{d^{i}}{d x^{i}} U_{j}\right)_{i=0, \ldots, 2 j-1 ; j=0, \ldots, l}(x), \mu\right)=G((\Gamma U)(x), \mu),
$$

where $G: \mathbb{R}^{n} \times \mathbb{R}^{p} \rightarrow \mathbb{R}^{k}$ is smooth. Here, $\mu \in \mathbb{R}^{p}$.

Owing to (4.2) and (4.3), the partial differential equation (4.1) is equivalent to a first order ordinary differential equation. Indeed, $u=\Gamma U$ solves

$$
u^{\prime}=C u-B G(u, \mu):=f(u, \mu)
$$

if and only if $U$ solves

$$
\mathcal{A} U+F(U, \mu)=0 .
$$

Suppose that $U_{1}$ is a primary pulse solution of (4.1) for $\mu=\mu_{1}$ with corresponding steady-state $\bar{U}$, see Section 2. Without loss of generality, we assume that the homogeneous equilibrium $\bar{U}=0$ is zero. Let $U_{N}$ denote an $N$-pulse relative to $U_{1}$. Then the linearizations of (4.1) at $U_{1}$ and $U_{N}$ are given by the densely defined closed operators

$$
\begin{aligned}
L_{1} & =\mathcal{A}+D_{U} F\left(U_{1}, \mu_{1}\right), \\
L_{N} & =\mathcal{A}+D_{U} F\left(U_{N}, \mu_{N}\right),
\end{aligned}
$$

on $B U\left(\mathbb{R}, \mathbb{R}^{k}\right)$, respectively. The eigenvalue problem for $L_{N}$ is given by

$$
L_{N} V=\left(\mathcal{A}+D_{U} F\left(U_{N}, \mu_{N}\right)\right) V=\lambda V .
$$

Let $q_{N}=\Gamma U_{N}$ be the $N$-homoclinic solution of (4.4) corresponding to $U_{N}$. Then $v=\Gamma V$ is a bounded solution of

$$
v^{\prime}=C v+B\left(\lambda-D_{u} G\left(q_{N}, \mu_{N}\right)\right) v+B H=\left(D_{u} f\left(q_{N}, \mu_{N}\right)+\lambda B\right) v+B H
$$

for $H \in B U\left(\mathbb{R}, \mathbb{R}^{k}\right)$ if and only if $V \in B U\left(\mathbb{R}, \mathbb{R}^{k}\right) \cap D(L)$ solves

$$
L_{N} V=\lambda V+H .
$$

Therefore, the approach given in Section 2 is applicable to equation (4.5) provided the hypotheses (H1) and (H2) stated there are satisfied for (4.4). In particular, Jordan chains

$$
\begin{aligned}
& \left(L_{N}-\lambda_{0}\right) P_{1, i}=0, \\
& \left(L_{N}-\lambda_{0}\right) P_{k, i}=P_{k-1, i}
\end{aligned}
$$


associated with the operator $L_{N}$ and the eigenvalue $\lambda_{0}$ are given by solutions $v_{k, i}$

$$
\begin{aligned}
& v_{1, i}^{\prime}=\left(D_{u} f\left(q_{N}, \mu_{N}\right)+\lambda_{0} B\right) v_{1, i}, \\
& v_{k, i}^{\prime}=\left(D_{u} f\left(q_{N}, \mu_{N}\right)+\lambda_{0} B\right) v_{k, i}+B v_{k-1, i}
\end{aligned}
$$

for $i=1, \ldots, m$ and $1<k \leq p(i)$. We suppose here that the geometric multiplicity of $\lambda_{0}$ is equal to $m$.

Owing to (H1), zero is not contained in the essential spectrum of the linearization of (4.1) evaluated at the trivial equilibrium $\bar{U}=0$. By [Hen81, Theorem A.1], the following alternative holds: either zero is not contained in the essential spectrum of the linearization $L_{1}$ of (4.1) evaluated at the primary pulse $U_{1}$ or there exists a $\delta>0$ such that any $\lambda \in U_{\delta}(0)$ is an eigenvalue of $L_{1}$. Indeed, $L_{1}$ is a relatively compact perturbation of the linearization at $\bar{U}$. This follows easily from ArzelaAscoli's theorem and the fact that $U_{1}(x)$ converges to zero for $|x|$ tending to infinity. The eigenvalues close to zero associated with $U_{1}$ coincide with zeroes of $E_{1}(\lambda)$ by Theorem 2 and the above discussion. Here, $E_{1}(\lambda)$ is the function described in Theorem 2 when applied to the primary pulse itself. Therefore, zero is contained in the essential spectrum of $L_{1}$ iff $E_{1}(\lambda)=0$ for $\lambda \in U_{\delta}(0)$. We will assume that zero is a normal point of the spectrum.

(H3) Zero is not contained in $\sigma_{e s s}\left(L_{1}\right)$.

Then $E_{1}(\lambda)$ is not identically equal to zero. By the remark in Section 3.3, we conclude that the function $E(\lambda)$ associated with the $N$-pulse $U_{N}$ does not vanish either. Hence, the essential spectrum of $L_{N}$ is bounded away from zero once (H3) is fulfilled. Therefore, the isolated zeroes of $E(\lambda)$ close to zero correspond to eigenvalues of $L_{N}$ with finite algebraic multiplicity and the remainder part of $\sigma\left(L_{N}\right)$ is bounded away from zero.

Lemma 4.1. Assume that (H1)-(H3) are fulfilled. Then $\lambda_{0} \in \sigma\left(L_{N}\right) \cap U_{\delta}(0)$ if and only if $E\left(\lambda_{0}\right)=0$. Moreover, mult $\left(\lambda_{0} ; L_{N}\right)=\operatorname{ord}\left(\lambda_{0} ; E\right)$; i.e., the algebraic multiplicity of the eigenvalue $\lambda_{0}$ of $L_{N}$ coincides with the order of $\lambda_{0}$ as a zero of $E(\lambda)$.

In [GJ90, Lemmata 5, 6] the multiplicity of the Evans function is related to the algebraic multiplicity of the eigenvalue. However, all attempts of the author to prove directly a relation between the Evans function investigated in [AGJ90, GJ90] to the function $E(\lambda)$ studied here failed.

Throughout, we fix an eigenvalue $\lambda_{0}$ of $L_{N}$ close to zero. Suppose that the structure of the generalized eigenspace associated with $\lambda_{0}$ is given by (4.7). Owing to the estimates of $W(\lambda)$ in Lemma 3.5, the operator $V(\lambda)=\mathcal{U}+W(\lambda)$ appearing in (3.58) is injective. Using this fact and Theorem 2 we conclude that there exist precisely $m$ linearly independent vectors $d_{1,1}, \ldots, d_{1, m} \in \mathbb{R}^{N}$ such that $S\left(\lambda_{0}\right) d_{1, i}=$ 0 and

$$
\Gamma P_{1, i}=V\left(\lambda_{0}\right) d_{1, i}
$$

for $i=1, \ldots, m$. Let $V(\lambda) d_{1, i}=: v_{1, i}(\lambda)$. In particular, we obtain $m=\operatorname{dim} \operatorname{Ke} S\left(\lambda_{0}\right)$. Next define

$$
w_{k, i}(\lambda):=\sum_{j=1}^{k} \frac{1}{(k-j) !} W^{(k-j)}(\lambda) d_{j, i}=\sum_{j=0}^{k-1} \frac{1}{j !} W^{(j)}(\lambda) d_{k-j, i},
$$


for $k \geq 2$, where we used the notation $W^{(j)}=\frac{d^{j}}{d \lambda^{j}} W$. Moreover, let $v_{k, i}(\lambda)$ be given by

$$
v_{k, i}(\lambda)=\mathcal{U} d_{k, i}+w_{k, i}(\lambda)
$$

see (3.58). Owing to the analyticity of $W(\lambda)$, the functions $w_{k, i}(\lambda)$ are well-defined.

Lemma 4.2. Let $p(i) \in \mathbb{N}$ for $i=1, \ldots, m$. The functions $v_{k, i}\left(\lambda_{0}\right)$ solve (4.8) for $i=1, \ldots, m$ and $1<k \leq p(i)$ if and only if the vectors $d_{k, i}$ solve

$$
\sum_{j=0}^{k-1} \frac{1}{j !} S^{(j)}\left(\lambda_{0}\right) d_{k-j, i}=0
$$

again for $i=1, \ldots, m$ and $1<k \leq p(i)$.

Proof. It is straightforward to calculate that $w_{k, i}(\lambda)$ solve

$$
\dot{w}_{k, i}^{ \pm}=D_{u} f\left(q^{ \pm}, \mu_{N}\right) w_{k, i}^{ \pm}+\left(G^{ \pm}(t)+\lambda B^{ \pm}(t)\right) w_{k, i}^{ \pm}+\lambda h^{ \pm} d_{k, i}+B v_{k-1, i}(\lambda)
$$

using (3.5) and the definition of $h^{ \pm}$in (3.8). The jumps $\xi_{k, i}=w_{k, i}^{+}(\lambda)(0)-w_{k, i}^{-}(\lambda)(0)$ are given by

$$
\xi_{k, i}=\sum_{j=0}^{k-1} \frac{1}{j !} S^{(j)}\left(\lambda_{0}\right) d_{k-j, i} .
$$

Hence the functions $w_{k, i}\left(\lambda_{0}\right)$ are generalized eigenfunctions if and only if all jumps vanish. This proves the lemma.

Proof. (Lemma 4.1). By the equivalence of (4.7) and (4.8), Lemma 4.2 reformulates the existence of Jordan chains for the operator $\left(L_{N}-\lambda_{0}\right)$ in terms of solutions of the system

$$
\sum_{j=0}^{k-1} \frac{1}{j !} S^{(j)}\left(\lambda_{0}\right) d_{k-j, i}=0 .
$$

Defining

$$
S_{j}:=\frac{1}{j !} S^{(j)}\left(\lambda_{0}\right)
$$

yields $S(\lambda)=\sum_{i=0}^{\infty} S_{j}\left(\lambda-\lambda_{0}\right)^{j}$, Computing solutions of (4.9) is therefore equivalent to the computation of the kernel of the matrix $\widetilde{S}_{M}$ given by

$$
\left(\widetilde{S}_{M}\right)_{i j}= \begin{cases}0, & i<j \leq M+1, \\ S_{i-j}, & j \leq i \leq M+1,\end{cases}
$$

for large $M$. An application of Lemma B.1 shows that the dimension of this kernel is equal to the order of $\operatorname{det} S(\lambda)$ at $\lambda=\lambda_{0}$. This proves the lemma.

\section{Calculating zeroes of $E(\lambda)$}

In this section, we give some general strategies on solving the reduced equations $(3.56)$

$$
\xi=(A-M \lambda+R(\lambda)) d=S(\lambda) d=0
$$


with respect to $\lambda \in \mathbb{C}$ close to zero and $d \in \mathbb{R}^{N} \backslash\{0\}$. Here, $A=\left(a_{i j}\right)$ is given by

$$
a_{i j}= \begin{cases}\left\langle\psi\left(T_{i}\right), \dot{q}^{-}\left(-T_{i}\right)\right\rangle, & j=i+1, \\ -\left\langle\psi\left(-T_{i-1}\right), \dot{q}^{+}\left(T_{i-1}\right)\right\rangle, & j=i-1, \\ \left\langle\psi\left(-T_{i-1}\right), \dot{q}^{+}\left(T_{i-1}\right)\right\rangle-\left\langle\psi\left(T_{i}\right), \dot{q}^{-}\left(-T_{i}\right)\right\rangle, & j=i, \\ 0, & \text { otherwise }\end{cases}
$$

for $i, j=1, \ldots, N$ and

$$
M=\int_{-\infty}^{\infty}\left\langle\psi_{1}(t), B\left(q_{1}(t), \mu_{1}\right) \dot{q}_{1}(t)\right\rangle d t \in \mathbb{R} .
$$

Remember that $T_{0}=T_{N}=\infty$ by definition and $T=\min _{j=1, \ldots, N-1} T_{j}$. Observe that the vector $d=(1, \ldots, 1)$ is contained in the kernel of $A$. The function $R(\lambda)$ defined in Lemma 3.6 is analytic in $\lambda$. Moreover, the estimate (3.57) holds; i.e., $R(\lambda)$ is small. Note that $a_{i j} \rightarrow 0$ as $T \rightarrow \infty$. We anticipate the expansion

$$
A(r)=r A_{0}+o(r)
$$

for some matrix $A_{0}$, where $r$ is related to $T$ by $r=r^{*}(T)$ for some function $r^{*}$ such that $r \rightarrow 0$ corresponds to $T \rightarrow \infty$. The matrix $A_{0}$ is independent of $r$ and thus of $T$. Such a scaling will frequently be possible in (5.1), see Section 6 .

Motivated by these facts, we investigate a linear equation of the form

$$
\left(r A_{0}-M \lambda+r A_{1}(r)+A_{2}(r, \lambda) \lambda\right) d=0
$$

for matrices $A_{0}, A_{1}(r)$ and $A_{2}(r, \lambda)$. Apparently, (5.4) admits a solution $(\lambda, d)$ with $d \neq 0$ if and only if the function

$$
E(r, \lambda):=\operatorname{det}(S(\lambda))=\operatorname{det}\left(r A_{0}-M \lambda+r A_{1}(r)+A_{2}(r, \lambda) \lambda\right)
$$

possesses a zero at $\lambda$. Throughout, we assume the following hypotheses.

(EV1) The spectrum of $A_{0}$ is given by $\sigma\left(A_{0}\right)=\left\{\nu_{1}, \ldots, \nu_{N-1}\right\} \cup\{0\}$ counted with multiplicity and $\nu_{j} \neq 0$ for $j=1, \ldots, N-1$.

(EV2) The matrices $A_{1}(r)$ and $A_{2}(r, \lambda)$ are continuous in $r \in\left[0, r_{0}\right]$ and fulfill $A_{1}(0)=A_{2}(0,0)=0$. Moreover, $A_{2}(r, \lambda)$ is analytic in $\lambda$.

(EV3) $M \neq 0$.

Scaling $\lambda$ by

$$
\lambda=r \nu
$$

we see that (5.4) is equivalent to

$$
\left(A_{0}-M \nu+A_{1}(r)+A_{2}(r, r \nu) \nu\right) d=:\left(A_{0}-M \nu+A_{3}(r, \nu)\right) d=0
$$

for $r \neq 0$. The matrix $A_{3}(r, \nu)$ is analytic in $\nu$ and satisfies

$$
A_{3}(r, \nu) \rightarrow 0 \text { as } r \rightarrow 0
$$

uniformly on compact subsets in $\nu$. Of course, (5.6) is equivalent to

$$
\widetilde{E}(r, \nu):=\operatorname{det}\left(A_{0}-M \nu+A_{3}(r, \nu)\right)=0,
$$

where $\widetilde{E}(r, \nu)$ is analytic in $\nu$. Define the set

$$
\mathcal{N}:=\left\{\frac{1}{M} \nu_{1}, \ldots, \frac{1}{M} \nu_{N-1}\right\}
$$

counted with multiplicity, that is, $\# \mathcal{N}=N-1$. Applying the Theorem of Rouché, we obtain the following lemma. 
Lemma 5.1. Assume (EV1)-(EV3). Then, for any $\eta>0$ with $\eta<\frac{1}{2} \operatorname{dist}(0, \mathcal{N})$, there exists an $r_{0}(\eta)>0$ with the following property. For any $r<r_{0}(\eta), \widetilde{E}(r, \cdot)$ possesses precisely $N-1$ zeroes in $U_{\eta}(\mathcal{N})$ counted with multiplicity and one zero in $U_{\eta}(0)$.

From the preceding discussion the next lemma is an immediate consequence of Lemma 5.1.

Lemma 5.2. Assume (EV1)-(EV3). Then there exists $r_{0}, \delta_{0}>0$ such that for any fixed $r \in\left[0, r_{0}\right], E(r, \cdot)$ possesses precisely $N$ zeroes in $U_{\delta_{0}}(0)$ counted with multiplicity. They are related to the zeroes of $\widetilde{E}(r, \nu)$ given in the previous lemma according to the transformation (5.5). In particular, their respective signs coincide.

Therefore, it is often sufficient to investigate the spectrum of the tridiagonal matrix $A_{0}$. Indeed, if zero is a simple eigenvalue of $A_{0}$ and the eigenvalues in $\mathcal{N}$ do not lie on the imaginary axis, the stability of the $N$-pulse is determined due to Lemmata 5.2 and 5.1. In applications, the matrix $A_{0}$ frequently possesses additional properties.

If the differential equation (3.2) is generic, the matrix $A_{0}$ often turns out to be triangular. In that case, the spectrum of $A_{0}$ is easily determined, because $A_{0}$ is already tridiagonal and hence the entries on the diagonal coincide with the eigenvalues.

Another interesting case arises, if the underlying differential equation (3.2) is time-reversible. Then $A_{0}$ is actually a symmetric tridiagonal matrix.

Lemma 5.3. Assume that there exists a linear involution $R: \mathbb{R}^{n} \rightarrow \mathbb{R}^{n}$ such that $f$ anticommutes with $R$, i.e., $f(R u, \mu)=-R f(u, \mu)$ for all $(u, \mu) \in \mathbb{R}^{n} \times \mathbb{R}^{p}$. Moreover, assume that $n=2 m$ is even and Fix $(R)=\mathbb{R}^{m} \times\{0\}$. Let $q_{1}(0) \in$ Fix $(R)$. Then the matrix A defined in (5.2) is symmetric.

Proof. Indeed, $q_{1}(0) \in \operatorname{Fix}(R)$ implies that $\dot{q}^{-}(-t)=-R \dot{q}^{+}(t)$ and $\psi(t)=R^{*} \psi(-t)$ for all $t \geq 0$. Therefore

$$
\begin{aligned}
a_{i, i-1} & =\left\langle\psi\left(-T_{i-1}\right), \dot{q}^{+}\left(T_{i-1}\right)\right\rangle=-\left\langle R^{*} \psi\left(T_{i-1}\right), R \dot{q}^{-}\left(-T_{i-1}\right)\right\rangle \\
& =-\left\langle\psi\left(T_{i-1}\right), \dot{q}^{-}\left(-T_{i-1}\right)\right\rangle=a_{i-1, i}
\end{aligned}
$$

and $A$ is symmetric.

While it seems difficult to express the eigenvalues of tridiagonal and symmetric matrices in terms of their coefficients, it is possible to determine their signs.

Lemma 5.4. Suppose that $A_{0}$ is a tridiagonal and symmetric $N \times N$ matrix. Assume that $(1, \ldots, 1)$ is contained in the kernel of $A_{0}$. Then $A_{0}=\left(a_{i j}^{0}\right)$ is determined by a vector $\left(a_{1}, \ldots, a_{N-1}\right) \in \mathbb{R}^{N-1}$ via

$$
a_{i j}^{0}= \begin{cases}-a_{i-1}, & j=i-1, \\ -a_{i}, & j=i+1 \\ a_{i}+a_{i-1}, & j=i \\ 0, & \text { otherwise }\end{cases}
$$


for $i, j=1, \ldots, N$ and with $a_{0}=a_{N}=0$. We define $\sigma\left(A_{0}\right)=:\{0\} \cup \tilde{\sigma}\left(A_{0}\right)$ counted with multiplicity. Then

$$
\begin{gathered}
\#\left(\tilde{\sigma}\left(A_{0}\right) \cap \mathbb{R}^{-}\right)=\#\left\{j \mid a_{j}<0\right\}, \\
\#\left(\tilde{\sigma}\left(A_{0}\right) \cap \mathbb{R}^{+}\right)=\#\left\{j \mid a_{j}>0\right\}, \\
\#\left(\tilde{\sigma}\left(A_{0}\right) \cap 0\right)=\#\left\{j \mid a_{j}=0\right\}
\end{gathered}
$$

obtains.

Lemma 5.4 is proved in Appendix C.

Next we give an interpretation of $M$ in terms of the Evans function $D(\lambda)$ associated with the primary pulse $q_{1}(t)$. This function is defined in the following way, see [AJ94, Section 2]. First, choose orientations $\left[E^{u}\right]$ and $\left[E^{s}\right]$ of the unstable and stable eigenspaces, such that the concatenation of these orients $\mathbb{R}^{n}$ in the canonical way. Then these orientations are transported to the tangent spaces of unstable and stable manifolds of

$$
\dot{u}=f(u, \mu)
$$

using the linearized flow. Define $E^{u}(\lambda)(t)$ and $E^{s}(\lambda)(t)$ as the unstable and stable manifolds of

$$
\dot{v}=\left(D_{u} f\left(q_{1}, \mu_{1}\right)+\lambda B\left(q_{1}, \mu_{1}\right)\right) v
$$

respectively. Then there exists unique volume forms $V^{u}(\lambda)$ and $V^{s}(\lambda)$ on $E^{u}(\lambda)(0)$ and $E^{s}(\lambda)(0)$, respectively, assigning the volume one to an orthonormal, oriented basis of these spaces. The Evans function is now defined by

$$
D(\lambda)=V^{u}(\lambda) \wedge V^{s}(\lambda) \in \mathbb{R} .
$$

Next, choose vectors $v_{1}^{u}, \ldots, v_{n_{u}-1}^{u}$ and $v_{1}^{s}, \ldots, v_{n_{s}-1}^{s}$ such that

$$
\begin{aligned}
& T_{q_{1}(0)} W^{u}\left(0, \mu_{1}\right)=\operatorname{span}\left(\dot{q}_{1}(0), v_{1}^{u}, \ldots, v_{n_{u}-1}^{u}\right), \\
& T_{q_{1}(0)} W^{s}\left(0, \mu_{1}\right)=\operatorname{span}\left(\dot{q}_{1}(0), v_{1}^{s}, \ldots, v_{n_{s}-1}^{s}\right),
\end{aligned}
$$

and

$$
\begin{aligned}
{\left[\dot{q}_{1}(0), v_{1}^{u}, \ldots, v_{n_{u}-1}^{u}\right] } & =\left[E^{u}\right], \\
{\left[\dot{q}_{1}(0), v_{1}^{s}, \ldots, v_{n_{s}-1}^{s}\right] } & =\left[E^{s}\right] .
\end{aligned}
$$

Lemma 5.5. The derivative of the Evans function associated with $q_{1}(t)$ is given by

$$
\begin{aligned}
& D^{\prime}(0)=\int_{-\infty}^{\infty}\left\langle\psi_{1}(t), B\left(q_{1}(t), \mu_{1}\right) \dot{q}_{1}(t)\right\rangle d t \\
& \quad \cdot \psi_{1}(0) \wedge v_{1}^{u} \wedge \cdots \wedge v_{n_{u}-1}^{u} \wedge \dot{q}_{1}(0) \wedge v_{1}^{s} \wedge \cdots \wedge v_{n_{s}-1}^{s} .
\end{aligned}
$$

Proof. The claim follows from [AGJ90, Section 2, (2.15) and (2.17)] and the results given in Section 3 applied to $q_{1}$, itself, i.e. for $N=1$. Indeed, then the jump is given by

$$
w_{1}^{-}(\lambda)(0)-w_{1}^{+}(\lambda)(0)=\left(\int_{-\infty}^{\infty}\left\langle\psi_{1}(t), B\left(q_{1}(t), \mu_{1}\right) \dot{q}_{1}(t)\right\rangle d t\right) \psi_{1}(0) \lambda+O\left(|\lambda|^{2}\right)
$$

and the derivative with respect to $\lambda$ yields the desired expression using [AGJ90, (2.17)] and the fact that volume forms are antisymmetric. 
Remark (i). If $\psi(0)$ is chosen such that

$$
\psi_{1}(0) \wedge v_{1}^{u} \wedge \cdots \wedge v_{n_{u}-1}^{u} \wedge \dot{q}_{1}(0) \wedge v_{1}^{s} \wedge \cdots \wedge v_{n_{s}-1}^{s}=1
$$

then

$$
D^{\prime}(0)=\int_{-\infty}^{\infty}\left\langle\psi_{1}(t), B\left(q_{1}(t), \mu_{1}\right) \dot{q}_{1}(t)\right\rangle d t=M,
$$

where $M$ already appeared in Theorem 2 . Here, an $n$-form in $\mathbb{R}^{n}$ is identified with $\mathbb{R}$ using the volume form distinguished by the orientation. For a one-dimensinal unstable manifold, i.e. $n_{u}=1$, this corresponds to a choice of $\psi_{1}$ such that

$$
\lim _{t \rightarrow \infty} e^{2 \alpha^{u} t}\left\langle\psi_{1}(t), q_{1}(-t)\right\rangle>0,
$$

where $\alpha^{u}$ denotes the unique unstable eigenvalue of $D_{u} f\left(0, \mu_{1}\right)$. Note that this condition coincides with the sign convention in [PW92, (1.3)], who proved Lemma 5.5 under the assumption of one-dimensional unstable manifolds, see [PW92, Theorem 1.11].

(ii) It turns out that

$$
D^{\prime}(0)>0
$$

holds along stable primary pulses in several applications. In particular, it arises in systems of reaction-diffusion equations, see [AGJ90, Proposition 2.2], and [AJ94, Lemma 4.2 including its proof], or for systems possessing one-dimensional unstable manifolds, see [PW92, Corollary 1.18].

In the next remark we indicate how a change of $\psi$ influences equation (5.1).

Remark (i). The time-reversed system.

Consider the systems

$$
\begin{aligned}
\frac{d}{d t} u & =f(u, \mu), \\
\frac{d}{d t} \tilde{u} & =-f(\tilde{u}, \mu)
\end{aligned}
$$

with associated $N$-pulses $u(t)=\tilde{u}(-t)$, matrices $A$ and $\widetilde{A}$, and Melnikov coefficients $M$ and $\widetilde{M}$, respectively. Then, due to $\frac{d}{d t} u(t)=-\frac{d}{d t} \tilde{u}(-t)$, it follows that $\sigma(A)=$ $-\sigma(\widetilde{A})$ and $M=-\widetilde{M}$.

(ii) Change of $\psi_{1}$.

Equation (5.1) is linear in $\psi_{1}$, whence a change of $\psi_{1}$ in $A_{0}$ or $M$ does not change the eigenvalues. However, changing $\psi_{1}$ to $-\psi_{1}$ in $A_{0}$ or exclusively in $M$ corresponds to multiplying the solutions $\nu$ of $\operatorname{det}\left(A_{0}-M \nu\right)=0$ by $(-1)$.

These simple remarks might be useful when applying the results in Section 6 in different particular equations.

\section{Applications}

In this section we apply the theory developed above to a particular bifurcation scenario, namely the saddle-focus bifurcation. Consider

$$
\begin{aligned}
& \dot{u}=f(u, \mu), \\
& \dot{v}=\left(D_{u} f(u, \mu)+\lambda B(u, \mu)\right) v
\end{aligned}
$$


for $u, v \in \mathbb{R}^{n}$ and $(\mu, \lambda) \in \mathbb{R} \times \mathbb{C}$ with $f, B \in C^{2}$. We assume that $\left(q_{1}, \mu_{1}\right)$ is a primary homoclinic solution of (6.1) converging to zero

$$
\lim _{t \rightarrow \pm \infty} q_{1}(t)=0
$$

Moreover, we assume the following hypothesis.

There are simple eigenvalues $-\alpha \pm i \beta_{0}$ for some $\alpha, \beta_{0}>0$ contained in $\sigma\left(D_{u} f\left(0, \mu_{1}\right)\right)$. The modulus of the real part of any other eigenvalue of $D_{u} f\left(0, \mu_{1}\right)$ is strictly larger than $\alpha$.

The homoclinic orbit $q_{1}(t)$ is supposed to be nondegenerate

$$
T_{q_{1}(0)} W^{s}\left(0, \mu_{1}\right) \cap T_{q_{1}(0)} W^{u}\left(0, \mu_{1}\right)=\mathbb{R} \dot{q}_{1}(0) .
$$

We denote by $\psi_{1}$ the bounded solution of

$$
\dot{w}=-D_{u} f\left(q_{1}, \mu_{1}\right)^{*} w,
$$

see Section 2. The next assumption is concerned with the asymptotic behaviour of $q_{1}$ and $\psi_{1}$.

(A3) The limits $\lim _{t \rightarrow \infty} e^{\alpha t}\left|q_{1}(t)\right| \neq 0$ and $\lim _{t \rightarrow-\infty} e^{-\alpha t}\left|\psi_{1}(t)\right| \neq 0$ are nonzero.

The assumptions (A2) and (A3) are generically fulfilled. Finally, we assume that $q_{1}(t)$ is constructed transversally and that the Evans function $D(\lambda)$ associated with the primary pulse $q_{1}$ is regular at $\lambda=0$, i.e. $D^{\prime}(0) \neq 0$. In other words,

$$
\int_{-\infty}^{\infty}\left\langle\psi_{1}(t), D_{\mu} f\left(q_{1}(t), \mu_{1}\right)\right\rangle d t=: M_{1} \neq 0
$$

and

$$
\int_{-\infty}^{\infty}\left\langle\psi_{1}(t), B\left(q_{1}(t), \mu_{1}\right) \dot{q}_{1}(t)\right\rangle d t=: M_{2} \neq 0
$$

are satisfied. We define the sets

$$
\begin{aligned}
& \mathcal{R}=\left\{\exp \left(-\frac{2 \pi \alpha}{\beta_{0}} n\right) \mid n \in \mathbb{N}_{0}\right\} \cup\{0\}, \\
& \mathcal{A}=\left\{\exp \left(-\frac{\pi \alpha}{\beta_{0}} k\right) \mid k \in \mathbb{N}_{0}\right\} .
\end{aligned}
$$

Note that $\mathcal{R}$ is a closed metric space. Then we can state the theorem.

Theorem 3. Assume that (A1)-(A5) are satisfied. Then there exists a $\delta>0$ such that for any $N \geq 2$ the following holds.

For any sequence $a_{j}^{0} \in \mathcal{A}$ for $j=1, \ldots, N-1$ with $a_{i}^{0} \in\left\{1, \exp \left(-\frac{\pi \alpha}{\beta_{0}}\right)\right\}$ for some $i$, there exists an $r_{0} \in \mathcal{R}, r_{0} \neq 0$ with the following properties.

(i) There are $C^{0}$-functions $a_{j}(r), \mu(r) \in \mathbb{R}$ for $r \in \mathcal{R}, r \leq r_{0}$ with $a_{j}(0)=$ $a_{j}^{0}$ for $j=1, \ldots, N-1$ and $\mu(0)=\mu_{1}$.

(ii) For any $r \in \mathcal{R}$ with $0<r \leq r_{0}$, there exists an $N$-pulse $q_{N}(r)$ for $\mu=\mu(r)$ with return times

$$
T_{j}(r)=-\frac{1}{\alpha} \ln \left(a_{j}(r) r\right)+\widetilde{T}, \quad j=1, \ldots, N-1,
$$

for some constant $\widetilde{T}$.

(iii) The $N$-pulses fulfilling (ii) are unique. 
Denote by $k_{j}^{0}$ the natural numbers associated with the $a_{j}^{0} \in \mathcal{A}$ chosen above. Then the stability of the $N$-pulses described above is determined as follows.

(iv) For $M_{2}>0\left(M_{2}<0\right)$, we have

$$
\begin{aligned}
& \#\{\lambda \in \mathbb{C}|E(\lambda)=0,| \lambda \mid<\delta, \operatorname{Re} \lambda<0\}=\#\left\{j \mid 1 \leq j \leq N-1, k_{j}^{0} \text { is even }(\text { odd })\right\}, \\
& \#\{\lambda \in \mathbb{C}|E(\lambda)=0,| \lambda \mid<\delta, \operatorname{Re} \lambda>0\}=\#\left\{j \mid 1 \leq j \leq N-1, k_{j}^{0} \text { is odd (even) }\right\},
\end{aligned}
$$

counted with multiplicity.

(v) $\lambda=0$ is a simple zero of $E(\lambda)$ and there are no other zeroes with norm less than $\delta$ on the imaginary axis.

The geometric interpretation of $k_{j}^{0}$ even or odd is given in Lemma 6.1, (6.4)(i) and (6.5), see also Figure 3.

We need some preparations before giving the proof of Theorem 3. First, we choose coordinates such that zero is an equilibrium of (6.1) for all $\mu$ close to $\mu_{1}$. Moreover, time is rescaled such that the real part of the simple eigenvalues $\alpha \pm i \beta(\mu)$ of $D_{u} f(0, \mu)$ is independent of $\mu$. Here, $\beta\left(\mu_{1}\right)=\beta_{0}$ holds. Afterwards, we change coordinates according to Lemmas 2.2 and 2.1. This does not change the stability properties of the $\mathrm{N}$-pulses provided (6.2) is transformed according to Lemma 2.3. Throughout, we assume that (A1)-(A5) are fulfilled.

Lemma 6.1. For t sufficiently large,

(i) $\left.\left\langle\psi(\mu)(-t), q^{+}(\mu)\right)(t)\right\rangle=s(\mu) e^{-2 \alpha t} \sin (2 \beta(\mu) t+\varphi(\mu))+O\left(e^{-(2 \alpha+\gamma) t}\right)$,

(ii) $\left\langle\psi(\mu)(-t), \dot{q}^{+}(\mu)(t)\right\rangle=s(\mu) e^{-2 \alpha t}(\beta(\mu) \cos (2 \beta(\mu) t+\varphi(\mu))$

$$
-\alpha \sin (2 \beta(\mu) t+\varphi(\mu)))+O\left(e^{-(2 \alpha+\gamma) t}\right),
$$

(iii) $\left|\left\langle\psi(\mu)(t), q^{-}(\mu)(-t)\right\rangle\right|+\left|\left\langle\psi(\mu)(t), \dot{q}^{-}(\mu)(-t)\right\rangle\right| \leq C e^{-(2 \alpha+\gamma) t}$

hold uniformly in $\mu$. Here, $\gamma>0$ is a constant. The functions $\varphi(\mu)$ and $s(\mu)$ are in $C^{2}$ and

$$
s_{0}:=s\left(\mu_{1}\right)>0 .
$$

Proof. The estimate (iii) follows from (A1). By [San93, Lemma 1.5] and (A1) there exist vectors $v(\mu)$ and $w(\mu) \in \mathbb{R}^{n}$ contained in the eigenspaces of the eigenvalues $(-\alpha \pm i \beta(\mu))$ of $D_{u} f(0, \mu)$ and $D_{u} f(0, \mu)^{*}$, respectively, such that

$$
\begin{aligned}
q^{+}(\mu)(t) & =\exp \left(D_{u} f(0, \mu) t\right) v(\mu)+O\left(e^{-(\alpha+\gamma) t}\right), \\
\psi(\mu)(-t) & =\exp \left(D_{u} f(0, \mu)^{*} t\right) w(\mu)+O\left(e^{-(\alpha+\gamma) t}\right)
\end{aligned}
$$

for $t \rightarrow \infty$. Moreover, $v(\mu)$ and $w(\mu)$ are smooth in $\mu$ and according to (A3) we conclude $v\left(\mu_{1}\right), w\left(\mu_{1}\right) \neq 0$. Using the expansion (6.6) and the fact that $v$ and $w$ are therefore nonzero eigenvectors of $(-\alpha \pm i \beta(\mu))$, it is straightforward - but lengthy to obtain (6.4)(i) and (ii).

Note that due to Lemma 6.1 we avoid a linearization of the flow near the equilibrium. In the next lemma, we formulate the equations for the return times $T_{j}$, solutions of which correspond to $N$-homoclinic solutions of (6.1) close to $q_{1}$. It turns out that the following definitions simplify the equations a lot. 


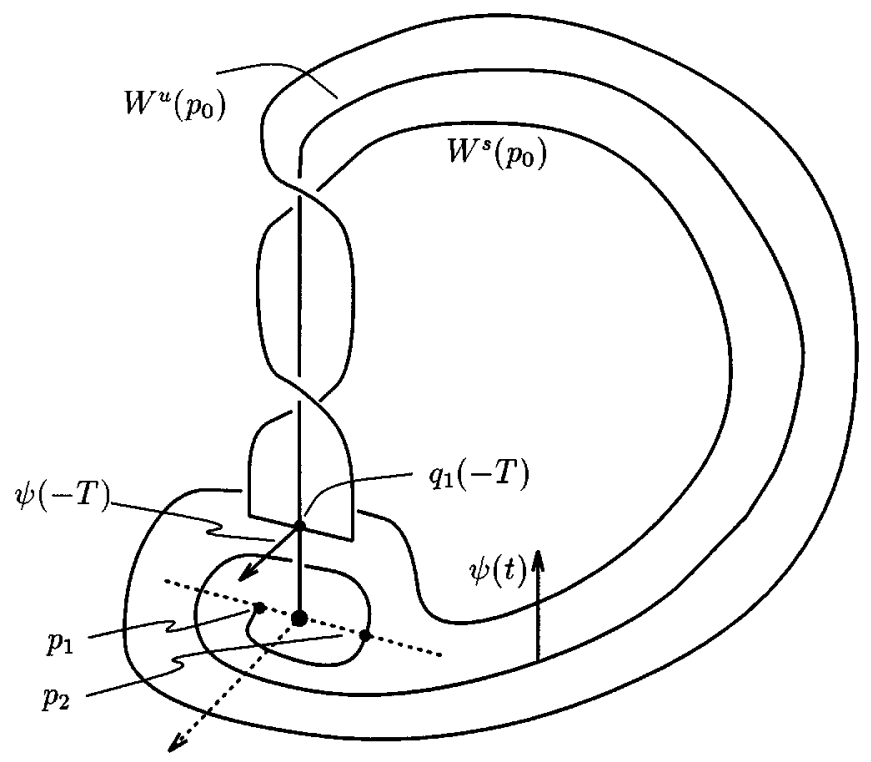

Figure 3. The geometry of the saddle-focus case: if $T$ is chosen such that $q(T)=p_{1}$ or $q(T)=p_{2}$, a 2-pulse exists with return time close to $T$. If $q(T)=p_{1}$ the 2-pulse is stable, while it is unstable if $q(T)=p_{2}$ (provided $M_{2}>0$ ).

For given large real numbers $T$ and $T_{j}, j=1, \ldots, N-1$, such that $T_{j} \geq T$ for all $j$ we define

$$
\begin{aligned}
r & =e^{-\alpha\left(2 T+\varphi_{0} / \beta_{0}\right)}, \quad T=-\frac{1}{2 \alpha} \ln r-\frac{\alpha \varphi_{0}}{\beta_{0}}, \\
a_{j} & =e^{-2 \alpha\left(T_{j}-T\right)}, \quad T_{j}=-\frac{1}{2 \alpha} \ln \left(a_{j} r\right)-\frac{\varphi_{0}}{2 \beta_{0}} .
\end{aligned}
$$

Here, $\varphi_{0}:=\varphi\left(\mu_{1}\right)$. Thus $r \in\left(0, \delta_{0}\right), a_{j} \in(0,1]$ for all $j$.

Lemma 6.2. Let $N \geq 2$ be fixed. Then there exists an $N$-pulse $\left(q_{N}, \mu\right)$ of (6.1) close to $\left(q_{1}, \mu_{1}\right)$ in phase space with associated return times $\left(T_{j}\right)_{j=1, \ldots, N-1}$ if and only if

$$
\begin{aligned}
a_{j} r e^{\frac{\varphi_{0}}{2 \beta_{0}}} \sin \left(-\frac{\beta(\mu)}{\alpha} \ln \left(a_{j} r\right)+\tilde{\varphi}(\mu)\right)+M_{1}\left(\mu-\mu_{1}\right) & =O\left(r^{1+\gamma}\right), \\
M_{1}\left(\mu-\mu_{1}\right) & =O\left(r^{1+\gamma}\right)
\end{aligned}
$$

for $j=1, \ldots, N-1$. The variables $a_{j}$ and $r$ are related to $T_{j}$ via (6.7). The function $\tilde{\varphi}$ is given by

$$
\tilde{\varphi}(\mu)=\frac{\varphi_{0}}{\beta_{0}} \beta(\mu)-\varphi(\mu)=O\left(\mu-\mu_{1}\right) .
$$

The remainder terms are smooth in $a_{j} \in(0,1]$ and $\mu$ up to $r=0$ and

$$
\frac{d}{d a_{j}} O=O\left(r^{1+\gamma}\right), \quad \frac{d}{d \mu} O=O\left(r^{1+\gamma}\right)
$$

holds. 
Proof. The jump equations for $N$-homoclinic solutions read

$$
\left\langle\psi\left(T_{i}\right), q^{-}\left(-T_{i}\right)\right\rangle-\left\langle\psi\left(-T_{i-1}\right), q^{+}\left(T_{i-1}\right)\right\rangle-M_{1}\left(\mu-\mu_{1}\right)+R\left(\left(T_{j}\right), \mu\right)=0,
$$

see [San93, Satz 3]. The claims follow now from the estimates of the remainder term given in [San93, Satz 3], Lemma 6.1 and (A4). For $a_{j} \in(0,1]$ the remainder terms are differentiable in $a_{j}$ and satisfy

$$
\frac{d}{d a_{j}} O=\frac{d}{d T_{j}} O \frac{d T_{j}}{d a_{j}}=O\left(e^{-2 \alpha T_{j}} e^{-\gamma T}\right) \frac{1}{a_{j} r} r=O\left(a_{j} r^{1+\gamma}\right) \frac{1}{a_{j}}=O\left(r^{1+\gamma}\right)
$$

uniformly in $r>0$ by using [San93, Satz 3]. Therefore, the remainder terms can be extended by zero to $r=0, a_{i} \in(0,1]$ in a differentiable way. This proves the lemma.

Now the reason for introducing the coordinates (6.7) is apparent. They allow for extending the bifurcation equations differentiably to $T_{j}=\infty$-at least if all of them tend to zero at the same order - and get rid of the trivial solution $r=0$ corresponding to the primary pulse $q_{1}$. In a certain sense, $r \rightarrow 0$ corresponds to a blow-up of the original bifurcation equation stated in [San93, Satz 3] by introduction the polar coordinate $r$.

Now we give the proof of the theorem.

Proof of Theorem 3. We will first prove the statements (i)-(iii), that is, the existence part. By Lemma 6.2 we have to solve the system (6.8)

$$
\begin{aligned}
a_{j} r e^{\frac{\varphi_{0}}{\beta_{0}}} \sin \left(-\frac{\beta(\mu)}{\alpha} \ln \left(a_{j} r\right)+\tilde{\varphi}(\mu)\right)+M_{1}\left(\mu-\mu_{1}\right)=O\left(r^{1+\gamma}\right), \\
M_{1}\left(\mu-\mu_{1}\right)=O\left(r^{1+\gamma}\right),
\end{aligned}
$$

for $j=1, \ldots, N-1$, where the remainder term satisfies (6.9). Owing to (A4) we have $M_{1} \neq 0$, whence the last equation can be solved with respect to $\mu$ yielding

$$
\mu=\mu^{*}\left(a_{1}, \ldots, a_{N-1}, r\right)=\mu(a, r)=\mu_{1}+O\left(r^{1+\gamma}\right) .
$$

Substituting $\mu$ into the remaining equations we obtain

$$
a_{j} r \sin \left(-\frac{\beta\left(\mu^{*}(a, r)\right)}{\alpha} \ln \left(a_{j} r\right)+\tilde{\varphi}\left(\mu^{*}(a, r)\right)\right)=O\left(r^{1+\gamma}\right), \quad j=1, \ldots, N-1 .
$$

Dividing by $r$ yields

$$
a_{j} r \sin \left(-\frac{\beta\left(\mu^{*}(a, r)\right)}{\alpha} \ln \left(a_{j} r\right)+\tilde{\varphi}\left(\mu^{*}(a, r)\right)\right)=O\left(r^{\gamma}\right), \quad j=1, \ldots, N-1
$$

Now, we have

$$
\begin{aligned}
\tilde{\varphi}\left(\mu^{*}\right) & =O\left(\mu^{*}-\mu_{1}\right)=O\left(r^{1+\gamma}\right), \\
\beta\left(\mu^{*}\right)-\beta_{0} & =O\left(\mu^{*}-\mu_{1}\right)=O\left(r^{1+\gamma}\right)
\end{aligned}
$$


in a $C^{1}$-sense with respect to $a$ by (6.10). Substituting this into the argument of the sine function, we obtain

$$
\begin{aligned}
\sin ( & \left.-\frac{\beta\left(\mu^{*}\right)}{\alpha} \ln \left(a_{j} r\right)+\tilde{\varphi}\left(\mu^{*}\right)\right) \\
& =\sin \left(-\frac{\beta_{0}}{\alpha} \ln \left(a_{j} r\right)-\frac{\beta\left(\mu^{*}\right)-\beta_{0}}{\alpha} \ln \left(a_{j} r\right)+\tilde{\varphi}\left(\mu^{*}\right)\right) \\
& =\sin \left(-\frac{\beta_{0}}{\alpha} \ln \left(a_{j} r\right)\right)+O\left(r^{1+\gamma}\right) .
\end{aligned}
$$

The estimate is valid for derivatives with respect to $a$, too. We restrict to $r \in \mathcal{R}$, whence $r=\exp \left(-\frac{2 \pi \alpha}{\beta_{0}} n\right)$ for some $n \in \mathbb{N}$ or $r=0$. Hence, (6.12) reads

$$
a_{j} \sin \left(-\frac{\beta_{0}}{\alpha} \ln a_{j}\right)=O\left(e^{-\gamma n}\right)
$$

for $j=1, \ldots, N-1$. The remainder term appearing in (6.15) is continuous in $(a, r)$ up to $r=0$ and partially differentiable with respect to $a_{i}$ again up to $r=0$ for $a_{j} \in(0,1]$ by $(6.9)$ and the arguments given above. Setting $r=0(n=\infty)$ in $(6.15)$ we obtain the equations

$$
a_{j} \sin \left(-\frac{\beta_{0}}{\alpha} \ln a_{j}\right)=0
$$

Therefore, the chosen sequence $\left(a_{j}^{0}\right)_{j=1, \ldots, N-1}$ with $a_{j}^{0} \in \mathcal{A}$ solves (6.16). In addition, the partial derivatives of $(6.16)$ with respect to $a_{j}$ evaluated at $a_{j}^{0}$ are given by

$$
\left.\left(\sin \left(-\frac{\beta_{0}}{\alpha} \ln a_{j}\right)-\frac{\beta_{0}}{\alpha} \cos \left(-\frac{\beta_{0}}{\alpha} \ln a_{j}\right)\right)\right|_{a_{j}=a_{j}^{0}=\exp \left(-\frac{\pi \alpha}{\beta_{0}} k_{j}^{0}\right)}=(-1)^{k_{j}^{0}+1} \frac{\beta_{0}}{\alpha}
$$

and thus are nonzero. All other entries in the Jacobian except the ones on the diagonal computed in (6.17) vanish.

By (6.16), (6.17) and the differentiability properties of (6.15) stated above, we can use the implicit function theorem to conclude that statements (i)-(iii) of the theorem are fulfilled.

It remains to prove items (iv) and (v), which are concerned with the stability properties of the $N$-pulses. By Theorem 2 we have to find zeroes of

$$
E(\lambda)=\operatorname{det}\left(A-M_{2} \lambda+R(\lambda)\right)
$$

We have the estimate

$$
\|R(\lambda)\|=O\left(r^{1+\gamma}+|\lambda|\left(r^{\gamma}+|\lambda|\right)\right)
$$

owing to Theorem 2, (A1) and (6.10). Moreover, by Lemma 6.1, we obtain

$$
A=r A_{1}(r)+O\left(r^{1+\gamma}\right)
$$


where $A_{1}(r)$ is the lower triangular, tridiagonal matrix

$$
\left(A_{1}\right)_{i j}= \begin{cases}0, & i=1, \\ s_{0} e^{\frac{\varphi_{0}}{2 \beta_{0}}} \beta_{0} \cos \left(-\frac{\beta_{0}}{\alpha} \ln a_{i}(r)\right), & j=i, i>1, \\ -s_{0} e^{\frac{\varphi_{0}}{2 \beta_{0}}} \beta_{0} \cos \left(-\frac{\beta_{0}}{\alpha} \ln a_{i}(r)\right), & j=i-1, i>1 \\ 0, & \text { otherwise }\end{cases}
$$

Indeed, the scalar products in the definition of $A$ given in Theorem 2 can be estimated by $(6.4)(\mathrm{i})$ and (ii). The sine term appearing in (6.4)(ii) is $O\left(r^{1+\gamma}\right)$ by (6.11), while the cosine is handled similarly to (6.14). Therefore, (6.19) obtains. Observe that for $r \rightarrow 0$ the limit

$$
\lim _{r \rightarrow 0} \cos \left(-\frac{\beta_{0}}{\alpha} \ln a_{i}(r)\right)=\cos \left(-\frac{\beta_{0}}{\alpha} \ln a_{i}^{0}\right)=\cos \left(-\pi k_{i}^{0}\right)=(-1)^{k_{i}^{0}}
$$

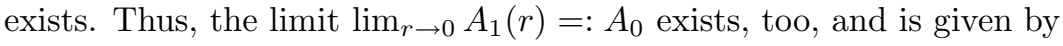

$$
\left(A_{0}\right)_{i j}= \begin{cases}0, & i=1 \\ (-1)^{k_{i}^{0}} s_{0} \beta_{0} e^{\frac{\varphi_{0}}{2 \beta_{0}}}, & j=i, i>1 \\ (-1)^{k_{i}^{0}+1} s_{0} \beta_{0} e^{\frac{\varphi_{0}}{2 \beta_{0}}}, & j=i-1, i>1 \\ 0, & \text { otherwise. }\end{cases}
$$

$A_{0}$ admits the eigenvalues

$$
\begin{aligned}
\nu_{j} & =(-1)^{k_{j}^{0}} s_{0} \beta_{0} e^{\frac{\varphi_{0}}{2 \beta_{0}}}, \quad j=1, \ldots, N-1, \\
\nu_{N} & =0 .
\end{aligned}
$$

Owing to (6.5) and (A1) the product $s_{0} \beta_{0}>0$ is positive.

Therefore, (6.18) reads

$$
E(\lambda)=\operatorname{det}\left(r A_{0}-M_{2} \lambda+r\left(A_{1}(r)-A_{0}\right)+O\left(r^{1+\gamma}\right)+O\left(|\lambda|\left(r^{\gamma}+|\lambda|\right)\right)\right)
$$

with $\left(A_{1}(r)-A_{0}\right)=o(1)$ and

$$
\sigma\left(A_{0}\right)=\{0\} \cup\left\{(-1)^{k_{j}^{0}} s_{0} \beta_{0} e^{\frac{\varphi_{0}}{2 \beta_{0}}} \mid j=1, \ldots, N-1\right\} .
$$

The statements (iv) and (v) follow now directly from Lemmata 5.1 and 5.2 for all $r \in \mathcal{R}$ sufficiently small by observing that (EV1)-(EV3) are fulfilled.

The proof of the theorem is therefore complete.

\section{Discussion}

First, let us compare our results to the ones obtained in [AJ93] for triple pulses. The statement of Theorem 3 is valid for the time-reversed system, too, if one takes the remark at the end of Section 5 into account. This clarifies a comment in [AJ93, p. 192].

We would like to emphasize that Theorem 2 (in combination with Lemma A.1) should be applicable to other bifurcations producing multiple pulses, too. In particular, the Hamiltonian and reversible saddle-focus bifurcations studied by [Dev76] and [Cha94, Här93], respectively, can be handled. Here, the Hamiltonian saddlefocus arises in the localized buckling of struts on a nonlinear elastic foundation describing their equilibrium states, see [BCT94]. The stability question is important (and unsolved) in this application. Moreover, the technique should work for some of the bifurcations to double pulses investigated first by [Yan87]. These are the 
nonorientable resonant bifurcation [CDF90], the inclination-flip [KKO93] and the orbit-flip [San93]. In the flip bifurcations, $N$-pulses for any $N$ occur if certain conditions on the eigenvalues are satisfied, see [HKK94] and [San93]. However, in these case, further improvements of the estimates stated in Theorem 2 and Lemma A.1 seem to be necessary in order to apply the technique presented here. A detailed investigation of the stability properties of pulses occurring in the bifurcations mentioned above is in progress.

The whole theory carries over to front and back solutions. Indeed, the existence results obtained in [Lin90] or [San93] are formulated for general heteroclinic cycles. It is possible to extend the stability results to this setting, too.

The reduced system

$$
E(\lambda)=\operatorname{det} S(\lambda)=\operatorname{det}(A-M \lambda i d+R(\lambda))=0
$$

given in Theorem 2 describes only the eigenvalues close to zero. Actually, $E(\lambda)$ and the Evans function $D(\lambda)$ do not coincide in general. Indeed, by construction, $E(\lambda)$ takes only changes of the $N$-pulses in the direction of $\psi(0)$ into account, but neglects the changes of the vectors $v_{i}^{u}$ and $v_{i}^{s}$ with $\lambda$, see Lemma 5.5 and [AJ94, Section 2]. However, using the technique presented here, it is possible to describe the splitting of other eigenvalues which are not necessarily close to zero. Instead of parametrizing the solutions of the eigenvalue equation by

$$
v^{ \pm}=\left(\dot{q}^{ \pm}+\dot{u}^{ \pm}\right) d+w^{ \pm}
$$

as in (3.5), one has to use coordinates according to

$$
v^{ \pm}=\varphi d+w^{ \pm},
$$

where $\varphi(t)$ is an eigenfunction with eigenvalue $\lambda_{0}$ of the primary pulse. Then one obtains a reduced system of the form

$$
\operatorname{det}\left(A-D^{\prime}\left(\lambda_{0}\right)\left(\lambda-\lambda_{0}\right) i d+R\left(\lambda-\lambda_{0}\right)\right)=0
$$

for $\lambda$ close to $\lambda_{0}$. Provided $\lambda_{0}$ is a simple zero of the Evans function, i.e. a simple eigenvalue, the reduced equation describes the splitting of precisely $N$ eigenvalues of the $N$-pulse close to $\lambda_{0}$. In fact, the principal part of the Evans function associated with an $N$-pulse can be computed nearby any eigenvalue of the primary pulse.

Frequently, travelling waves are investigated in the context of singular perturbed equations like the FitzHugh-Nagumo equation. It would be interesting to compare the matrix $S(\lambda)$ appearing in the reduced equation

$$
E(\lambda)=\operatorname{det} S(\lambda)=0
$$

to the SLEP-matrix, see [Nis94] and the references therein. A relation between the SLEP matrix and the Evans function was detected in [SNI94]. While the method studied here is described in terms of ordinary differential equations, the SLEP method takes a partial-differential-equation-point of view.

\section{A. Improved estimates}

In this appendix, we give improved estimates for the jumps $\xi$. They are needed when investigating $N$-pulses bifurcating from codimension-two points. Suppose that the following hypothesis is fulfilled. 
(P) There exist projections $Q_{+}^{u}(t)$ and $Q_{+}^{u u}(t)$ for $t>0$ such that

$$
\begin{array}{rlrl}
P_{+}^{u}(t) & =Q_{+}^{u}(t)+Q_{+}^{u u}(t), & & \left|\Phi_{+}(s, t) Q_{+}^{u}(t)\right| \leq C e^{-\alpha^{u}(t-s)}, \\
Q_{+}^{u}(t) Q_{+}^{u u}(t) & =Q_{+}^{u u}(t) Q_{+}^{u}(t)=0, & \left|\Phi_{+}(s, t) Q_{+}^{u u}(t)\right| \leq C e^{-\alpha^{u u}(t-s)}
\end{array}
$$

is satisfied for some $0<\alpha^{u}<\alpha^{u u}$ and $0<s<t$. In addition, $P_{0}^{u}=Q_{0}^{u}+Q_{0}^{u u}$ for spectral projections $Q_{0}^{u}$ and $Q_{0}^{u u}$. We assume that $\lim _{t \rightarrow \infty} Q_{+}^{u}(t)=Q_{0}^{u}$ and $\lim _{t \rightarrow \infty} Q_{+}^{u u}(t)=Q_{0}^{u u}$. Moreover, the same holds for the stable projection $P_{-}^{s}(t)$ for $t<0$.

We define

$$
\begin{aligned}
p_{1}(T):=\sup _{t \geq T}\left(\left|P_{+}^{s}(t)+Q_{+}^{u}(t)-P_{0}^{s}-Q_{0}^{u}\right|+\left|Q_{+}^{u u}(t)-Q_{0}^{u u}\right|\right. \\
\left.\quad+\left|P_{-}^{u}(-t)+Q_{-}^{s}(-t)-P_{0}^{u}-Q_{0}^{s}\right|+\left|Q_{-}^{s s}(-t)-Q_{0}^{s s}\right|\right) .
\end{aligned}
$$

Although hypothesis $(\mathrm{P})$ might sound exotic it occurs in the investigation of the homoclinic flip-bifurcations, see [San93].

First we have

$$
\begin{aligned}
& \left|\Phi_{+}^{u}\left(0, T_{j}\right) P_{0}^{u} D_{j} d-\Phi_{+}^{u}\left(0, T_{j}\right) Q_{0}^{u} D_{j} d\right| \\
& \quad=\left|\Phi_{+}^{u}\left(0, T_{j}\right)\left(Q_{+}^{u}\left(T_{j}\right)+Q_{+}^{u u}\left(T_{j}\right)\right) P_{0}^{u} D_{j} d-\Phi_{+}^{u}\left(0, T_{j}\right) Q_{0}^{u} D_{j} d\right| \\
& \quad \leq\left|\Phi_{+}^{u}\left(0, T_{j}\right)\left(Q_{+}^{u}\left(T_{j}\right)-Q_{0}^{u}\right)+\Phi_{+}^{u}\left(0, T_{j}\right) Q_{+}^{u u}\left(T_{j}\right)\right|\left|P_{0}^{u} D_{j} d\right| \\
& \quad \leq C\left(e^{-\alpha T} p_{1}(T)+e^{-\alpha^{u u}} T\right)|D||d|
\end{aligned}
$$

by using $(\mathrm{P})$. Indeed, we have

$$
\begin{aligned}
& \Phi_{+}^{u}\left(0, T_{j}\right)\left(Q_{+}^{u}\left(T_{j}\right)-Q_{0}^{u}\right) P_{0}^{u} x \\
& \quad=\Phi_{+}^{u}\left(0, T_{j}\right)\left(Q_{+}^{u}\left(T_{j}\right)-Q_{0}^{u}\right) P_{0}^{u} x+\Phi_{+}^{u}\left(0, T_{j}\right)\left(P_{+}^{s}(t)-P_{0}^{s}\right) P_{0}^{u} x
\end{aligned}
$$

owing to $\Phi_{+}^{u}\left(0, T_{j}\right)=\Phi_{+}^{u}\left(0, T_{j}\right) P_{+}^{u}(t)$ and Lemma 3.2. By the estimate (3.48),

$$
\begin{aligned}
\left|\Phi_{+}^{u}\left(0, T_{j}\right) a_{j}^{+}\right|= & \left|\Phi_{+}^{u}\left(0, T_{j}\right)\left(P_{0}^{u} D_{j} d+\left(A_{2}(\lambda)(b, d)\right)_{j}^{+}\right)\right| \\
\leq & \left|\Phi_{+}^{u}\left(0, T_{j}\right) P_{0}^{u} D_{j} d\right|+C\left(e^{-\alpha T}+|G|+|\lambda|\right)|b| \\
& \quad+C\left(p_{1}(T)+|G|\right)|D||d|+C|\lambda||d| \\
\leq & C\left(e^{-\alpha T}\left|Q_{0}^{u} D\right|+\left(p_{1}(T)+|G|+e^{-\alpha^{u u} T}\right)|D|+|\lambda|\right)|d| \\
& \quad+C\left(e^{-\alpha T}+|G|+|\lambda|\right)|b|
\end{aligned}
$$

obtains. Of course, the corresponding estimate holds for $\left|\Phi_{-}^{s}\left(0,-T_{j}\right) a_{j}^{-}\right|$. Therefore, owing to (3.40), we conclude the improved estimate

$$
\begin{aligned}
& \left|L_{4}(\lambda)\right| \leq \delta|b|+\left|\Phi_{+}^{u}\left(0, T_{j}\right) a_{j}^{+}\right|+\left|\Phi_{-}^{s}\left(0,-T_{j-1}\right) a_{j-1}^{-}\right| \\
& \quad+C((|G|+|\lambda|)|w|+|\lambda||d|) \\
& \leq \delta|b|+C\left(e^{-\alpha T}\left(\left|Q_{0}^{u} D\right|+\left|Q_{0}^{s} D\right|\right)\right. \\
& \left.\quad+\left(p_{1}(T)+|G|+e^{-\alpha^{u u} T}+e^{-\alpha^{s s} T}\right)|D|+|\lambda|\right)|d|
\end{aligned}
$$

replacing (3.42). Thus, the estimate (3.44) can be sharpened yielding

$$
\begin{aligned}
\left|B_{1}(\lambda) d\right| \leq C & \left(e^{-\alpha T}\left(\left|Q_{0}^{u} D\right|+\left|Q_{0}^{s} D\right|\right)\right. \\
& \left.+\left(p_{1}(T)+|G|+e^{-\alpha^{u u} T}+e^{-\alpha^{s s}} T\right)|D|+|\lambda|\right)|d| .
\end{aligned}
$$


Next we reformulate equation (3.27)

$$
\begin{aligned}
P_{-}^{s}\left(-T_{j}\right) a_{j}^{-}-P_{+}^{u}\left(T_{j}\right) a_{j}^{+}=D_{j} d+\Phi_{+}^{s}\left(T_{j}, 0\right) b_{j}^{+}-\Phi_{-}^{u}\left(-T_{j}, 0\right) b_{j+1}^{-} \\
\quad+\int_{0}^{T_{j}} \Phi_{+}^{s}\left(T_{j}, s\right)\left(\left(G_{j}^{+}(s)+\lambda B_{j}^{+}(s)\right) w_{j}^{+}(s)+\lambda h_{j}^{+}(s) d_{j}\right) d s \\
\quad+\int_{-T_{j}}^{0} \Phi_{-}^{u}\left(-T_{j}, s\right)\left(\left(G_{j+1}^{-}(s)+\lambda B_{j+1}^{-}(s)\right) w_{j+1}^{-}(s)+\lambda h_{j+1}^{-}(s) d_{j+1}\right) d s \\
=: D_{j} d+\left(L_{5}(\lambda)(b, d, w)\right)_{j}
\end{aligned}
$$

with

$$
\left|L_{5}(\lambda)(b, d, w)\right| \leq C\left(\left(e^{-\alpha T}+|G|+|\lambda|\right)|b|+(|G|+|\lambda|)|a|+|\lambda||d|\right),
$$

see (3.29). Applying the projections $P_{0}^{u}$ and $P_{0}^{s}$ yields

$$
\begin{aligned}
P_{-}^{s}\left(-T_{j}\right) a_{j}^{-}= & P_{0}^{s} D_{j} d+P_{0}^{s}\left(P_{+}^{u}\left(T_{j}\right)-P_{0}^{u}\right) a_{j}^{+}+P_{0}^{u}\left(P_{-}^{s}\left(-T_{j}\right)-P_{0}^{s}\right) a_{j}^{-} \\
& +P_{0}^{s}\left(L_{5}(\lambda)(b, d, w)\right)_{j}, \\
P_{+}^{u}\left(T_{j}\right) a_{j}^{+}=- & P_{0}^{u} D_{j} d-P_{0}^{u}\left(P_{-}^{s}\left(-T_{j}\right)-P_{0}^{s}\right) a_{j}^{-}+P_{0}^{s}\left(P_{+}^{u}\left(T_{j}\right)-P_{0}^{u}\right) a_{j}^{+} \\
& -P_{0}^{u}\left(L_{5}(\lambda)(b, d, w)\right)_{j} .
\end{aligned}
$$

Using the definition

$$
p_{2}(T):=\sup _{t \geq T}\left(\left|P_{0}^{s}\left(P_{+}^{u}(t)-P_{0}^{u}\right)\right|+\left|P_{0}^{u}\left(P_{-}^{s}(-t)-P_{0}^{s}\right)\right|\right),
$$

and (3.37) and (A.4) for estimating $|a|$ and $|b|$, respectively, we obtain

$$
\begin{aligned}
\mid P_{-}^{s}( & \left.-T_{j}\right) a_{j}^{-}-P_{0}^{s} D_{j} d|+| P_{+}^{u}\left(T_{j}\right) a_{j}^{+}+P_{0}^{u} D_{j} d \mid \\
& \leq p_{2}(T)|a|+\left|L_{5}(\lambda)(b, d, w)\right| \\
& \leq C\left(p_{2}(T)|a|+\left(e^{-\alpha T}+|G|+|\lambda|\right)|b|+(|G|+|\lambda|)|a|+|\lambda||d|\right) \\
& \leq C\left(\left(e^{-\alpha T} p_{1}(T)+p_{2}(T)+|G|+e^{-\alpha^{u u} T}+e^{-\alpha^{s s} T}\right)|D|\right. \\
& \left.\quad+e^{-2 \alpha T}\left(\left|Q_{0}^{u} D\right|+\left|Q_{0}^{s} D\right|\right)+|\lambda|\right)|d| \\
& \leq C(|\lambda|+S)|d| .
\end{aligned}
$$

Here, $S$ is defined by

$S:=\left(\left(e^{-\alpha T} p_{1}(T)+p_{2}(T)+|G|+e^{-\alpha^{u u} T}+e^{-\alpha^{s s} T}\right)|D|+e^{-2 \alpha T}\left(\left|Q_{0}^{u} D\right|+\left|Q_{0}^{s} D\right|\right)\right)$,

where $p_{1}$ and $p_{2}$ have been given in (A.1) and (A.6), respectively.

Lemma A.1. Assume hypothesis (P). Then the jumps $\xi$ satisfy

$\xi_{i}=\left\langle\psi\left(T_{i}\right), P_{0}^{u} D_{i} d\right\rangle+\left\langle\psi\left(-T_{i-1}\right), P_{0}^{s} D_{i-1} d\right\rangle-\lambda \int_{-\infty}^{\infty}\langle\psi(t), H(t)\rangle d t d_{i}+(R(\lambda) d)_{i}$

and $R(\lambda)$ satisfies the improved estimate

$$
|R(\lambda) d| \leq C\left(e^{-\alpha T} S+|G|^{2}|D|+\left(e^{-\alpha T}+|G|+\left\|h_{i}-H\right\|+|\lambda|\right)|\lambda|\right)|d|,
$$

where $S$ is defined in (A.8). 
Proof. We refer here to Lemma 3.6. The estimate (3.49) can be replaced by

$$
\begin{aligned}
\left\langle\psi\left(T_{i}\right),\right. & \left.a_{i}^{+}\right\rangle-\left\langle\psi\left(-T_{i-1}\right), a_{i-1}^{-}\right\rangle \\
= & \left\langle\psi\left(T_{i}\right), P_{0}^{u} D_{i} d\right\rangle+\left\langle\psi\left(-T_{i-1}\right), P_{0}^{s} D_{i-1} d\right\rangle \\
& +\left\langle\psi(0), \Phi_{+}^{u}\left(0, T_{i}\right)\left(P_{+}^{u}\left(T_{i}\right) a_{i}^{+}+P_{0}^{u} D_{i} d\right)\right. \\
& \left.-\Phi_{-}^{s}\left(0,-T_{i-1}\right)\left(P_{-}^{s}\left(-T_{i-1}\right) a_{i-1}^{-}-P_{0}^{s} D_{i-1} d\right)\right\rangle \\
= & \left\langle\psi\left(T_{i}\right), P_{0}^{u} D_{i} d\right\rangle+\left\langle\psi\left(-T_{i-1}\right), P_{0}^{s} D_{i-1} d\right\rangle+O\left(e^{-\alpha T}(|\lambda|+S)|d|\right)
\end{aligned}
$$

due to (3.48) and (A.7). The other estimates in the proof of (3.47) remain unchanged and (A.9) obtains.

Now the estimates from Lemma 3.1 can be substituted as in Section 3.3.

\section{B. The multiplicity Lemma}

For any analytic function $V(\lambda)$ with values in the vector space of $N \times N$ matrices, we associate matrices $V_{j}$ for $j \geq 0$ by

$$
V(\lambda)=\sum_{j=0}^{\infty} \lambda^{j} V_{j}
$$

Using the Taylor coefficients $V_{j}$, we define the matrix $\widetilde{V}$

$$
\widetilde{V}_{i j}:= \begin{cases}0, & i<j \\ V_{i-j}, & i \geq j\end{cases}
$$

for $i, j \in \mathbb{N}_{0}$. Hence, the entries on each band of the matrix $\widetilde{V}$ coincide and the upper right triangle consists of zeroes

$$
\widetilde{V}=\left(\begin{array}{cccc}
V_{0} & & & 0 \\
V_{1} & V_{0} & & \\
V_{2} & V_{1} & V_{0} & \\
\vdots & \ddots & \ddots & \ddots
\end{array}\right)
$$

It is straightforward to check that the mapping $V(\lambda) \mapsto \widetilde{V}(\lambda)$ is an embedding of the algebra of analytic, matrix-valued functions to the algebra of matrices of the form (B.1). In particular, $\widetilde{V W}=\widetilde{V} \cdot \widetilde{W}$ holds.

The truncated matrix $\widetilde{V}_{M}$ for $M \in \mathbb{N}$ is given by the $(M+1) \times(M+1)$ matrix

$$
\left(\widetilde{V}_{M}\right)_{i j}= \begin{cases}0, & i<j \leq M+1, \\ V_{i-j}, & j \leq i \leq M+1 .\end{cases}
$$

We denote the order of a zero of an analytic, complex-valued function $f(\lambda)$ at $\lambda=\lambda_{0}$ by $\operatorname{ord}\left(\lambda_{0}, f\right)$. Moreover, Ke $\widetilde{V}_{M}$ denotes the kernel of $\widetilde{V}_{M}$.

Lemma B.1. Assume that $S(\lambda)$ is an analytic, matrix-valued function and suppose that $\operatorname{ord}(0, \operatorname{det} S)<\infty$. Then there exists an $M_{0}$ such that

$$
\operatorname{dim} \operatorname{Ke} \widetilde{S}_{M}=\operatorname{ord}(0, \operatorname{det} S(\lambda))
$$

for all $M \geq M_{0}$. 
Proof. Choose any analytic, matrix-valued functions $V(\lambda)$ and $W(\lambda)$ such that $V(0)$ and $W(0)$ are invertible. Observe that neither $\operatorname{ord}(0, \operatorname{det} S(\lambda))$ nor the dimension of the kernel of $\widetilde{S}_{M}$ changes if we replace $S(\lambda)$ by

$$
V(\lambda) S(\lambda) W(\lambda)
$$

and accordingly $\widetilde{S}_{M}$ by

$$
\widetilde{V}_{M} \widetilde{S}_{M} \widetilde{W}_{M}
$$

Indeed, the matrices $\widetilde{V}_{M}$ and $\widetilde{W}_{M}$ are invertible, because $V_{0}=V(0)$ and $W_{0}$ are.

This allows for transforming $S(\lambda)$ into a simpler form. First transform $S(0)$ such that

$$
S(0)=S_{0}=\left(\begin{array}{ll}
0 & 0 \\
0 & \underbrace{1}_{k}
\end{array}\right)
$$

for some $0 \leq k \leq N$ afterwards. Here we denote by $k$ the number of rows and columns of the identity appearing in the matrix. Let $n_{0}^{+}:=N-k$ and $n_{0}^{0}:=k$. Then

$$
S(\lambda)=\left(\begin{array}{cc}
\lambda A(\lambda) & \lambda B(\lambda) \\
\lambda C(\lambda) & 1+\lambda D(\lambda)
\end{array}\right)
$$

for some matrix-valued, analytic functions $A, B, C$ and $D$. Next let

$$
\begin{aligned}
V(\lambda) & =\left(\begin{array}{cc}
1 & -\lambda B(\lambda)(1+\lambda D(\lambda))^{-1} \\
0 & (1+\lambda D(\lambda))^{-1}
\end{array}\right), \\
W(\lambda) & =\left(\begin{array}{cc}
1 & 0 \\
-\lambda(1+\lambda D(\lambda))^{-1} C(\lambda) & 1
\end{array}\right)
\end{aligned}
$$

and transform $S(\lambda)$ according to (B.2). This yields the matrix function (which we again refer to as $S(\lambda)$ )

$$
S(\lambda)=\left(\begin{array}{cc}
\lambda\left(A(\lambda)-\lambda B(\lambda)(1+\lambda D(\lambda))^{-1} C(\lambda)\right) & 0 \\
0 & 1
\end{array}\right)=:\left(\begin{array}{cc}
\lambda T(\lambda) & 0 \\
0 & 1
\end{array}\right) .
$$

Now we can inductively perform the same procedure on $T(\lambda)$ as before on $S(\lambda)$. Therefore, after the $m$ th step, $S(\lambda)$ can be written according to

$$
S(\lambda)=\sum_{j=0}^{m} \lambda^{j} S_{j}+\lambda^{m+1} T_{m+1}(\lambda) .
$$

Here

$$
S_{j}=\left(\begin{array}{ccc}
0 & 0 & 0 \\
0 & 1 & 0 \\
0 & \underbrace{0}_{n_{j}^{+}} & \underbrace{0}_{n_{j}^{-}}
\end{array}\right)
$$

for $j=0, \ldots, m$ with

$$
n_{j}^{-}=\sum_{i=1}^{j-1} n_{i}^{0}, \quad n_{j}^{+}+n_{j}^{0}+n_{j}^{-}=N,
$$


and

$$
T_{m+1}(\lambda)=\left(\begin{array}{cc}
\underbrace{t_{m+1}(\lambda)}_{m+1} & 0 \\
\underbrace{0}_{n_{m}^{+}} & \underbrace{0}_{N-n_{m}^{+}}
\end{array}\right) .
$$

Thus the matrix $S(\lambda)$ looks like

$$
S(\lambda)=\left(\begin{array}{cccc}
\lambda^{m+1} t_{m+1}(\lambda) & & & 0 \\
& \lambda^{m} \cdot 1 & & \\
0 & & \ddots & \\
0 & & & 1
\end{array}\right),
$$

where some of the blocks might be of zero size. The determinant of $S(\lambda)$ up to products with functions $f(\lambda)$ such that $f(0) \neq 0$ is therefore given by

$$
\operatorname{det} S(\lambda)=\lambda^{m+1} \operatorname{det}\left(t_{m+1}(\lambda)\right) \cdot \prod_{j=1}^{m} \lambda^{j n_{j}^{0}} .
$$

In particular, the procedure described above has to stop at the $M$ th step, given by

$$
\sum_{i=1}^{M} n_{i}^{0}=N
$$

Otherwise, $\operatorname{det} t_{M+1}(\lambda)$ and therefore $\operatorname{det} S(\lambda)$ would be of infinite order at $\lambda=0$ contradicting the assumption. Hence

$$
S(\lambda)=\sum_{j=0}^{M} \lambda^{j} S_{j}, \quad \operatorname{det} S(\lambda)=\prod_{j=1}^{M} \lambda^{j n_{j}^{0}}
$$

for matrices $S_{j}$ given by (B.5), whence

$$
\operatorname{ord}(0, \operatorname{det} S(\lambda))=\sum_{j=1}^{M} j n_{j}^{0} .
$$

Next we compute the dimension of the kernel of the matrices $\widetilde{S}_{m}$ for $m \geq M$. Remember that the matrices $V(\lambda)$ and $W(\lambda)$ transform $S(\lambda)$ into the normal form (B.8) associated with the family $S_{j}$ defined in (B.5). The matrices $\widetilde{V}$ and $\widetilde{W}$ will transform the matrix $\widetilde{S}$ such that the transformed matrix is again given by the family $S_{j}$. Indeed, as mentioned above, the mapping $S \rightarrow \widetilde{S}$ is an injective algebrahomomorphism. Therefore, we can compute the dimension of the kernel of $\widetilde{S}_{m}$ using the matrices $S_{j}$ as defined in (B.5). Hence

$$
\widetilde{S}_{m}=\left(\begin{array}{cccc}
S_{0} & & & 0 \\
S_{1} & S_{0} & & \\
\vdots & \ddots & \ddots & \\
S_{m} & \ldots & S_{1} & S_{0}
\end{array}\right)
$$

for $m \geq M$ with $S_{j}=0$ for $j>M$ and

$$
\operatorname{dim} \operatorname{Ke} \widetilde{S}_{m}=\sum_{j=0}^{M} n_{j}^{+} .
$$


It remains to prove that the expressions in (B.9) and (B.10) coincide. Owing to (B.6) and (B.7) we obtain

$$
\begin{aligned}
\sum_{j=0}^{M} n_{j}^{+} & =\sum_{j=0}^{M}\left(N-n_{j}^{0}-n_{j}^{-}\right)=\sum_{j=0}^{M}\left(N-n_{j}^{0}-\sum_{i=0}^{j-1} n_{i}^{0}\right) \\
= & \sum_{j=0}^{M}\left(N-\sum_{i=0}^{j} n_{i}^{0}\right)=\sum_{j=0}^{M} \sum_{i=j+1}^{M} n_{i}^{0}=\sum_{j=0}^{M} j n_{i}^{0}
\end{aligned}
$$

and the lemma is proved.

\section{Proof of Lemma 5.4}

The matrix $A_{0}$ is symmetric, whence it leaves $V=(\mathbb{R}(1, \ldots, 1))^{\perp}$ invariant. The subspace $V$ admits the basis

$$
v_{j}=(0, \ldots, 0,1,-1,0, \ldots, 0) \text { for } j=1, \ldots, N-1,
$$

where the one is placed at the $j$ th component. Moreover, it is easy to see that

$$
A_{0} v_{j}=-a_{j-1} v_{j-1}+2 a_{j} v_{j}-a_{j+1} v_{j+1}
$$

for $j=1, \ldots, N-1$, where $a_{0}=a_{N}=0$ by definition. Hence, $A_{0}: V \rightarrow V$ is represented by the matrix

$$
B_{N-1}=\left(\begin{array}{ccccc}
2 a_{1} & -a_{1} & & & \\
-a_{2} & 2 a_{2} & -a_{2} & & \\
& \ddots & \ddots & \ddots & \\
& & -a_{N-2} & 2 a_{N-2} & -a_{N-2} \\
& & & -a_{N-1} & 2 a_{N-1}
\end{array}\right)
$$

with respect to the basis $\left(v_{j}\right)$ of $V$. We denote the matrix representation of $\left.A\right|_{V}$ by $B_{N-1}: \mathbb{R}^{N-1} \rightarrow \mathbb{R}^{N-1}$. The determinant of $B_{N}$ is given by

$$
\operatorname{det} B_{N}=\operatorname{det}\left(\begin{array}{ccccc}
2 a_{1} & 0 & & & \\
-a_{2} & \frac{3}{2} a_{2} & 0 & & \\
& \ddots & \ddots & \ddots & \\
& & -a_{N-1} & \frac{N}{N-1} a_{N-1} & 0 \\
& & & -a_{N} & \frac{N+1}{N} a_{N}
\end{array}\right)=(N+1) \prod_{i=1}^{N} a_{i} .
$$

Indeed, this can be proved by induction. First add one half of the first column to the second one, yielding a diagonal entry $\frac{3}{2}$ in the second column. Then notice that adding $\frac{i}{i+1}$ times the $i$ th column to the $(i+1)$ th one removes the element $b_{i, i+1}$ but changes the diagonal element $b_{i+1, i+1}$ to $\left(2-\frac{i}{i+1}\right)=\frac{i+2}{i+1}$. This proves the claim.

After this preparation, we prove the statements of Lemma 5.4 by induction on $N$. Therefore, suppose that Lemma 5.4 is proved for the matrices $B_{n}$ with $n \leq N-1$ and consider $B_{N}$ next. There are two different cases. 
(1) Suppose that $a_{k}=0$ for some $k$ with $1 \leq k \leq N$. The one row of $B_{N}$ vanishes identically, see (C.1). Moreover,

$$
B_{N}=\left(\begin{array}{cccccccc}
2 a_{1} & -a_{1} & & & & & & \\
-a_{2} & 2 a_{2} & -a_{2} & & & & & \\
& \ddots & \ddots & \ddots & & & & \\
& & -a_{k-1} & 2 a_{k-1} & -a_{k-1} & & & \\
& & & 0 & 0 & 0 & & \\
& & & & -a_{k+1} & 2 a_{k+1} & -a_{k+1} & \\
& & & & & \ddots & \ddots & \ddots \\
& & & & & & -a_{N} & 2 a_{N}
\end{array}\right) .
$$

Hence, there is one eigenvalue equal to zero, because the $k$ th row vanishes, while the other eigenvalues are determined by the two matrices $B_{k-1}\left(a_{1}, \ldots, a_{k-1}\right)$ and $B_{N-k}\left(a_{k+1}, \ldots, a_{N}\right)$ of size smaller than $N$. Using the induction step proves the claim in this case.

(2) Owing to (1), we can assume that $a_{i} \neq 0$ for $i=1, \ldots, N$. Define $\tilde{a}=$ $\left(a_{1}, \ldots, a_{N-1}\right)$ and consider $\hat{a}=(\tilde{a}, \varepsilon)$ for $\varepsilon \in \mathbb{R}$. Then the eigenvalues $\nu_{1}(\varepsilon), \ldots$, $\nu_{N}(\varepsilon)$ of $B_{N}(\tilde{a}, \varepsilon)$ are $C^{1}$ in $\varepsilon$ for fixed $\tilde{a}$. Indeed, by definition, they coincide with the eigenvalues of the symmetric matrix $A_{0}(\tilde{a}, \varepsilon)$, to which we can apply [Kat66, Theorem II.6.8].

Now, we take $\varepsilon=0$ first. Then, due to $a_{i} \neq 0$ for $i=1, \ldots, N-1$, we know that

$$
\nu_{1}(0), \ldots, \nu_{N-1}(0) \neq 0
$$

using (1). In particular, the claim follows for the eigenvalues $\nu_{1}(0), \ldots, \nu_{N-1}(0)$. Moreover,

$$
\prod_{i=1}^{N-1} \nu_{i}(0)=N \prod_{i=1}^{N-1} a_{i} \neq 0
$$

owing to (C.2) and

$$
\nu_{N}(0)=0,
$$

because the last row of $B_{N}(\tilde{a}, 0)$ vanishes. Therefore,

$$
\operatorname{sign}\left(\prod_{i=1}^{N-1} \nu_{i}(\varepsilon)\right)=\operatorname{sign}\left(\prod_{i=1}^{N-1} a_{i}\right) \neq 0
$$

for all small $\varepsilon$. Applying (C.2) to $B_{N}(\tilde{a}, \varepsilon)$ yields

$$
\prod_{i=1}^{N} \nu_{i}(\varepsilon)=(N+1) \varepsilon \prod_{j=1}^{N-1} a_{j} \neq 0
$$

and (C.3) implies that

$$
\operatorname{sign}\left(\nu_{N}(\varepsilon)\right)=\operatorname{sign}(\varepsilon)
$$

for all small $\varepsilon$. On the other hand,

$$
\operatorname{det} B_{N}(\tilde{a}, \varepsilon)=(N+1) \varepsilon \prod_{i=1}^{N-1} a_{i} \neq 0
$$

is valid for all $\varepsilon \neq 0$, whence no eigenvalue can change sign as long as $\varepsilon$ is nonzero. This proves the lemma. 
It is also possible to prove the lemma using [Wil65, paragraphs 36-38].

\section{REFERENCES}

[AGJ90] J. C. Alexander, R. A. Gardner, and C. K. R. T. Jones, A topological invariant arising in the stability analysis of travelling waves, J. Reine Angew. Math. 410 (1990), 167-212. MR 92d:58028

[AJ93] J. C. Alexander and C. K. R. T. Jones, Existence and stability of asymptotically oscillatory triple pulses, Z. Angew. Math. Phys. 44 (1993), 189-200. MR 94k:35042

[AJ94] , Existence and stability of asymptotically oscillatory double pulses, J. Reine Angew. Math. 446 (1994), 49-79. MR 94m:35152

[BCT94] B. Buffoni, A. R. Champneys, and J. F. Toland, Bifurcation and coalescence of a plethora of homoclinic orbits for a Hamiltonian system, J. Dynam. Differential Equations 8 (1996), 221-279. CMP 96:12

[BJ89] P. W. Bates and C. K. R. T. Jones, Invariant manifolds for semilinear partial differential equations. In Dynamics Reported (U. Kirchgraber and H.-O. Walther, editors), volume 2, pages 1-38, John Wiley \& Sons and Teubner, 1989. MR 90g:58017

[CDF90] S.-N. Chow, B. Deng, and B. Fiedler, Homoclinic bifurcation at resonant eigenvalues, J. Dyn. Diff. Eqs. 2 (1990), 177-244. MR 91g:58199

[Cha94] A. R. Champneys, Subsidiary homoclinic orbits to a saddle-focus for reversible systems, Int. J. Bifurcation and Chaos 4 (1994), 1447-1482. MR 96e:34074

[Dev76] R. L. Devaney, Homoclinic orbits in Hamiltonian systems, J. Diff. Eq. 21 (1976), 431438. MR 56:1365

[EFF82] J. W. Evans, N. Fenichel, and J. A. Feroe, Double impulse solutions in nerve axon equations, SIAM J. Appl. Math. 42 (1982), 219-234. MR 83h:92018a

[EMS90] C. Elphick, E. Meron, and E. A. Spiegel, Patterns of propagating pulses, SIAM J. Appl. Math. 50 (1990), 490-503. MR 91k:35033

[Fer86] J. A. Feroe, Existence of travelling wave trains in nerve axon equations, SIAM J. Appl. Math. 46 (1986), 1079-1097. MR 88e:92008

[Gas83] P. Gaspard, Generation of a countable set of homoclinic flows through bifurcation, Phys. Lett. A 97 (1983), 1-4. MR 84k:58186

[GJ90] R. A. Gardner and C. K. R. T. Jones, Traveling waves of a perturbed diffusion equation arising in a phase field model, Indiana Univ. Math. J. 39 (1990), 1197-1222. MR 92d:35039

[Gle89] P. Glendinning, Subsidiary bifurcations near bifocal homoclinic orbits, Math. Proc. Cambridge Phil. Soc. 105 (1989), 597-605. MR 90e:58118

[Här93] J. Härterich, Kaskaden homokliner Orbits in reversiblen dynamischen Systemen, Diploma thesis, University of Stuttgart, 1993.

[Hen81] D. Henry, Geometric theory of semilinear parabolic equations, Lecture Notes in Math. 840, Springer, New York, Berlin, Heidelberg, 1981. MR 83j:35084

[HKK94] A. J. Homburg, H. Kokubu, and M. Krupa, The cusp horseshoe and its bifurcations from inclination-flip homoclinic orbits, Ergodic Theory and Dynamical Systems 14 (1994), 667-693. MR 96a:58134

[Kat66] T. Kato, Perturbation Theory for Linear Operators, Springer-Verlag, Berlin, 1966. MR 34:3324

[KKO93] M. Kisaka, H. Kokubu, and H. Oka, Bifurcation to $N$-homoclinic orbits and N-periodic orbits in vector fields, J. Dyn. Diff. Eqs. 5 (1993), 305-358. MR 94m:58158

[Lin90] X.-B. Lin, Using Melnikov's method to solve Silnikov's problems, Proc. Royal Soc. Edinburgh 116A (1990), 295-325. MR 92b:58195

[Mer92] E. Meron, Pattern formation in excitable media, Physics Reports 218 (1992), 1-66. CMP 93:01

[Nii95a] S. Nii, An extension of the stability index for travelling wave solutions and its application for bifurcations, SIAM J. Math. Anal. 28 (1997), 402-433. CMP 97:08

[Nii95b] Stability of the travelling $N$-front $(N$-back) wave solutions of the FitzHughNagumo equations, SIAM J. Math. Anal. 28 (1997), 1094-1112.

[Nis94] Y. Nishiura, Coexistence of infinitely many stable solutions to reaction-diffusion systems in the singular limit, In Dynamics Reported (C. K. R. T. Jones, U. Kirchgraber, and H.-O. Walther, editors), volume 3, pages 25-103, Springer, New Series, 1994. 
[PW92] R. L. Pego and M. I. Weinstein, A class of eigenvalue problems, with applications to instabilities of solitary waves, Phil. Trans. Roy. Soc. London A 340 (1992), 47-94. MR 93g:35115

[San93] B. Sandstede, Verzweigungstheorie homokliner Verdopplungen, Doctoral thesis, University of Stuttgart, 1993.

[SNI94] H. Suzuki, Y. Nishiura, and H. Ikeda, Stability of traveling waves and a relation between the Evans function and the SLEP method, J. Reine Angew. Math. 475 (1996), 1-37. MR 97f:35014

[Wil65] J. H. Wilkinson, The algebraic eigenvalue problem, Clarendon Press, Oxford, 1965. MR 89j:65031

[Yan87] E. Yanagida, Branching of double pulse solutions from single pulse solutions in nerve axon equations, J. Diff. Eq. 66 (1987), 243-262. MR 88c:35078

[YM89] E. Yanagida and K. Maginu, Stability of double-pulse solutions in nerve axon equations, SIAM J. Appl. Math. 49 (1989), 1158-1173. MR 91d:35110

Weierstrass-Institut für Angewandte Analysis und Stochastik, Mohrenstrasse 39, 10117 Berlin, Germany

Current address: Department of Mathematics, The Ohio State University, Columbus, Ohio 43210-1174

E-mail address: sandstede@wias-berlin.de 